\title{
WPO, COV and IIA bargaining solutions for non-convex bargaining problems
}

\author{
Hans Peters · Dries Vermeulen
}

Accepted: 15 June 2010 / Published online: 1 July 2010

(C) The Author(s) 2010. This article is published with open access at Springerlink.com

\begin{abstract}
We characterize all $n$-person multi-valued bargaining solutions, defined on the domain of all finite bargaining problems, and satisfying Weak Pareto Optimality (WPO), Covariance (COV), and Independence of Irrelevant Alternatives (IIA). We show that these solutions are obtained by iteratively maximizing nonsymmetric Nash products and determining the final set of points by so-called LDR decompositions. If, next, we assume the (set-theoretic) Axiom of Determinacy, then this class coincides with the class of iterated Nash bargaining solutions; but if we assume the Axiom of Choice then we are able to construct an additional large set of discontinuous and even nonmeasurable solutions. We show however that none of these nonmeasurable solutions can be defined in terms of set theoretic formulae. We next show that a number of existing results in the literature as well as some new results are implied by our approach. These include a characterization of all WPO, COV and IIA solutionsincluding single-valued ones-on the domain of all compact bargaining problems, and an extension of a theorem of Birkhoff characterizing translation invariant and homogeneous orderings.
\end{abstract}

Keywords Nash bargaining solutions · Non-convex bargaining problems

JEL Classification $\quad$ C72 $\cdot$ D44

\footnotetext{
H. Peters · D. Vermeulen $(\varangle)$

Department of Quantitative Economics, Universiteit Maastricht, P. O. Box 616, 6200 MD Maastricht, The Netherlands

e-mail: d.vermeulen@maastrichtuniversity.nl

H. Peters

e-mail: h.peters@maastrichtuniversity.nl
} 


\section{Introduction}

In this paper we study $n$-person multi-valued bargaining solutions satisfying the well-known (cf. Nash 1950) axioms of Weak Pareto Optimality (WPO), Covariance (COV), and Independence of Irrelevant Alternatives (IIA), on domains of compact bargaining problems containing all finite problems.

The work of Nash (1950) focuses on the domain of convex bargaining problems. The restriction of bargaining solutions to convex bargaining problems is usually motivated by the possibility to randomize over outcomes, in combination with the assumption that players are expected utility maximizers. However, as among others Zhou (1996) already observes, these arguments are not compelling for several reasons. Randomization might not always be an option, for example because it may simply not be possible, or, even though theoretically possible and academically appealing, individuals concerned might not deem randomization desirable or even acceptable as part of the decision mechanism. Also, as has repeatedly been argued convincingly by many authorities in the field of utility theory, individuals need not have von NeumannMorgenstern utility functions. (See for example Machina (1982) or Starmer (2000) for an overview.) In fact, the empirical evidence that humans do not use expected utility to make decisions is overwhelming, see for example the seminal paper by Kahneman and Tversky (1979) and related literature.

Thus, there is a need to extend the classical results of Nash (1950) to domains of bargaining problems where convexity is no longer assumed. Since we allow for non-convex bargaining problems, some caution is needed in justifying the Covariance condition. The usual interpretation of this axiom, associated with the use of von Neumann-Morgenstern utility functions, would imply convexity of the bargaining problems under consideration. Here, to motivate the requirement of Covariance, we just assume that the preferences of the bargainers are uniquely represented up to positive linear transformations. ${ }^{1}$ Allowing for multi-valuedness of solutions is a natural consequence when considering non-convex bargaining problems.

We also do not impose continuity as a basic requirement. Adding continuity in general excludes the possibility to select from multi-valued Nash bargaining solutions. Many interesting solutions for non-convex bargaining problems, such as for example serial dictatorship solutions, ${ }^{2}$ would consequently be ruled out by insistence on continuity of the solution. In some interesting environments continuity even leads to an impossibility result. In particular, there is no single-valued continuous solution satisfying the three conditions WPO, COV and IIA on the domain of compact bargaining

\footnotetext{
1 We note that also in so-called non-expected utility theories representing utility functions are usually unique up to positive linear transformations, cf. Denicolò and Mariotti (2000). Moreover, it is possible to justify expected utility without all lotteries being available, cf. Fishburn (1972). Nevertheless, these are only partial justifications for considering the combination of covariance of solutions and non-convexity of bargaining problems.

2 A serial dictatorship solution is a bargaining solution in which first the first dictator chooses from the initial set of outcomes those outcomes that maximize his personal utility. Next, from the remaining set of outcomes, the second dictator chooses those that maximize the second dictator's utility. And so on. This is in fact a special case of an iterated Nash bargaining solution.
} 
problems. ${ }^{3}$ On the other hand, without continuity there is generally a large class of solutions additional to the continuous case, such as for example the serial dictatorship solutions, albeit that for some other additional ones we need the Axiom of Choice to define them, see Sect. 4.

Our basic results are the three characterizations presented in Sect. 3 of the paper, where we consider bargaining solutions defined on the domain of finite bargaining problems. The first result says that any WPO, COV and IIA bargaining solution $\varphi$ must be a refinement of some (nonsymmetric) Nash bargaining solution. More precisely, there is a nonzero nonnegative vector $\alpha \in \mathbb{R}^{n}$ such that each point $x$ assigned by $\varphi$ maximizes the product $\Pi_{i} x_{i}^{\alpha_{i}}$ on the bargaining problem under consideration. The level sets of such a ('Nash') product are called 'generalized indifference curves'. If we denote the generalized indifference curve through the vector $e=(1,1, \ldots, 1)$ by $I_{\varphi}$, the second result says that not only does each WPO, COV and IIA $\varphi$ induce a partition of $I_{\varphi}$ into three sets $L, D$, and $R$ with specific properties, but also that each such partition induces a different $\varphi$. These partitions are called LDR decompositions. The set $L$ in this decomposition consists of those points $x$ such that from the bargaining problem $\{x, e\}$ only the point $x$ is chosen by $\varphi$. Similarly, if only $e$ is chosen then $x \in R$, and if both $x$ and $e$ are chosen then $x \in D$. Together with the Nash products these LDR decompositions completely characterize the class of WPO, $\mathrm{COV}$, and IIA bargaining solutions. The third result takes this characterization considerably further and shows that each WPO, COV, and IIA bargaining solution can also be described by first iteratively maximizing Nash products and in the end applying an LDR decomposition.

In Sect. 4 we show that this last characterization is as far as we can go without making additional assumptions. In fact, we show that essentially opposite results are obtained depending on the basic set-theoretic axiom we wish to adopt. Under the so-called Axiom of Determinacy - which, in particular, implies that every subset of the interval $[0,1]$ is measurable-we obtain that every WPO, COV, and IIA bargaining solution is an iterated Nash bargaining solution. Under the Axiom of Choice (or, equivalently, Zorn's Lemma) we are able to construct (very) many other-in particular non-measurable-WPO, COV, and IIA bargaining solutions.

From a theoretical point of view there seems to be no particular reason to favor one of these basic axioms over the other. From a practical point of view however, even though the Axiom of Choice allows for many more solutions, some of which might conceivably be singled out by appealing axioms (we do not pursue this avenue here), these solutions are difficult to use exactly because they can only be obtained using the Axiom of Choice. ${ }^{4}$ Consequently these "extra" solutions, the ones besides the iterated Nash bargaining solutions, cannot be calculated in any concrete sense, and are therefore not computable beyond the mere observation "they exist". This point of view is substantiated in the last part of Sect. 4, where we show that none of these

\footnotetext{
3 This will be a consequence of Theorems 5.4 and 5.2 in this paper.

4 Karlis Podnieks writes on his website (Podnieks 2007): “...And the Platonist "world of sets" possesses some features of a mirage: it seems to promise large amounts of water in the middle of a desert, but does not keep the promise as you try to follow the vision".
} 
non-measurable solutions can be defined when only using elementary set-theoretic formulae in the definition.

Section 5 discusses a number of consequences. Adding (a weak form of) continuity results in a characterization of all (nonsymmetric) Nash bargaining solutions on any domain containing the finite domain. Adding symmetry instead of continuity results in a characterization of the symmetric $n$-person Nash bargaining solution. This basically implies a result by Mariotti (1998a), who characterizes the $n$-person symmetric Nash bargaining solution on the domain of finite bargaining problems, and also the recent result of $\mathrm{Xu}$ and Yoshihara (2006), characterizing the symmetric Nash bargaining solution on any domain of compact bargaining problems containing the domain of finite problems. ${ }^{5}$

The final result in Sect. 5 states that on the domain of all compact ${ }^{6}$ problems $^{-}$ WPO, COV and IIA characterize the set of iterated Nash bargaining solutions. This result — which is also derived in a different way by Naumova and Yanovskaya (2001), see below-not only implies but also refines the main results in Zhou (1996) and Denicolò and Mariotti (2000), which state that under a similar set of conditions plus single-valuedness, a solution selects from the maximizers of a Nash product. Here, we show how exactly this selection has to be made.

In Sect. 6 we show how our results imply a generalization of a theorem of Birkhoff (1948), which states that an ordering on $\mathbb{R}^{n}$ is translation invariant and positive homogeneous if and only if it maximizes a sequence of weighted sums with orthogonal weight vectors. We characterize the orderings that are obtained by dropping homogeneity, in terms of LDR compositions. ${ }^{7}$ It follows, moreover, that in Birkhoff's theorem homogeneity can be replaced by the Axiom of Determinacy. Naumova and Yanovskaya (2001) use Birkhoff's theorem to derive the characterization of iterated Nash bargaining solutions mentioned before. ${ }^{8}$ We could have started this paper from Birkhoff's theorem as well, but since our approach and results are more general, essentially we would have had to redo the proof of that theorem to obtain the generalization that we need. Therefore, the present paper starts from scratch and the generalization of Birkhoff's theorem follows as a byproduct.

A further discussion of related literature is deferred to the relevant places in the paper and also to Sect. 7. We start with preliminaries in Sect. 2. Some of the proofs in the paper are collected in a few appendices.

\section{Preliminaries}

For $x, y \in \mathbb{R}^{n}, x \leq y$ means $x_{i} \leq y_{i}$ for all $i$, and $x<y$ means $x_{i}<y_{i}$ for all $i$. The vector in $\mathbb{R}^{n}$ of which all coordinates are zero is denoted by 0 . The vector in

\footnotetext{
5 It is easy to check that for our results to go through it is sufficient to include, at least, all finitely generated comprehensive bargaining problems instead of the domain of finite problems.

6 This is for convenience, the result also holds for suitable subsets of this domain.

7 Birkhoff's Theorem is formulated for the additive case but there is a one-to-one correspondence between the additive case and the multiplicative case in our framework.

${ }^{8}$ In their paper, they consider social welfare orderings instead of choice functions.
} 
$\mathbb{R}^{n}$ of which all coordinates are one is denoted by $e$. The set of vectors $x$ in $\mathbb{R}^{n}$ with $x \geq 0$ is denoted by $\mathbb{R}_{+}^{n}$, and $\mathbb{R}_{++}^{n}$ denotes the set of vectors $x$ in $\mathbb{R}^{n}$ with $x>0$. For $a, x \in \mathbb{R}_{++}^{n}, a x:=\left(a_{1} x_{1}, \ldots, a_{n} x_{n}\right)$ and $\frac{x}{a}:=\left(\frac{x_{1}}{a_{1}}, \ldots, \frac{x_{n}}{a_{n}}\right)$. For $a \in \mathbb{R}^{n}$ and $S \subset \mathbb{R}^{n}, a S:=\{a x \mid x \in S\}$. We use the notation $S \subset T$ to indicate that $S$ is a subset of $T$, so $S=T$ is allowed. For a set $S \subset \mathbb{R}^{n}$, the closure of $S$ is denoted by $\bar{S}$.

A bargaining problem is a compact and non-empty set $B \subset \mathbb{R}_{++}^{n}$. (We implicitly assume the 'disagreement point' to be the origin.) A non-empty set $\mathcal{D}$ of bargaining problems is called a domain. In this paper we mainly consider the domain

$$
\mathcal{F}:=\left\{F \subset \mathbb{R}_{++}^{n} \mid F \text { is finite and not empty }\right\}
$$

of all finite $n$-person bargaining problems. The domain of all $n$-person bargaining problems is denoted by $\mathcal{C}$. Most other domains $\mathcal{D}$ we consider in this paper include $\mathcal{F}$, so $\mathcal{F} \subset \mathcal{D} \subset \mathcal{C}$.

A correspondence $\varphi: \mathcal{D} \rightarrow \mathbb{R}^{n}$ is called a (bargaining) solution if $\phi \neq \varphi(B) \subset B$ holds for all $B \in \mathcal{D}$. If specific elements, say $x$ and $y$ or $x^{1}, x^{2}$ and $x^{3}$ of $\mathbb{R}_{++}^{n}$ are involved, we typically write $\varphi(x, y)$ and $\varphi\left(x^{1}, x^{2}, x^{3}\right)$ instead of $\varphi(\{x, y\})$ and $\varphi\left(\left\{x^{1}, x^{2}, x^{3}\right\}\right)$.

A bargaining solution $\varphi: \mathcal{D} \rightarrow \mathbb{R}^{n}$ is a refinement of a bargaining solution $\psi: \mathcal{D} \rightarrow$ $\mathbb{R}^{n}$ if $\varphi(B) \subset \psi(B)$ for all $B \in \mathcal{D}$.

We define several properties that a bargaining solution $\varphi$ may have.

\section{WPO (Weak Pareto Optimality)}

For every $B \in \mathcal{D}$ and all $x, y \in B, x<y$ implies that $x \notin \varphi(B)$.

\section{PO (Pareto Optimality)}

For every $B \in \mathcal{D}$ and all $x, y \in B, x \leq y$ and $x \neq y$ implies that $x \notin \varphi(B)$.

IIA (Independence of Irrelevant Alternatives)

For all $B, C \in \mathcal{D}$ with $C \subset B$ and $C \cap \varphi(B) \neq \phi$ we have $\varphi(C)=C \cap \varphi(B)$.

COV (Covariance)

For all $a \in \mathbb{R}_{++}^{n}$ and all $B \in \mathcal{D}$ such that $a B \in \mathcal{D}, \varphi(a B)=a \varphi(B)$.

\section{CON (Continuity)}

Let $x^{k}, y^{k} \in \mathbb{R}_{++}^{n}$ for all $k \in \mathbb{N}, x^{k} \rightarrow x \in \mathbb{R}_{++}^{n}$ and $y^{k} \rightarrow y \in \mathbb{R}_{++}^{n}$. If $\left\{x^{k}, y^{k}\right\},\{x, y\} \in \mathcal{D}$ and $x^{k} \in \varphi\left(x^{k}, y^{k}\right)$ for all $k \in \mathbb{N}$, then $x \in \varphi(x, y)$.

\section{SYM (Symmetry)}

Let $B \in \mathcal{D}$ be symmetric, i.e., $B$ is invariant under any permutation of coordinates. If $x \in \varphi(B)$ and $y \in B$ can be obtained from $x$ by permuting its coordinates, then $y \in \varphi(B)$.

The properties of WPO, IIA, COV, and SYM were introduced by Nash (1950) for single-valued solutions. ${ }^{9}$

Let $\varphi: \mathcal{C} \rightarrow \mathbb{R}^{n}$ and $\psi: \mathcal{C} \rightarrow \mathbb{R}^{n}$ be two bargaining solutions. Suppose that $\varphi(C) \in$ $\mathcal{C}$ for all $C \in \mathcal{C}$. Then the composite bargaining solution $\psi \circ \varphi: \mathcal{C} \rightarrow \mathbb{R}^{n}$ is defined by, for all $C \in \mathcal{C}$,

\footnotetext{
${ }^{9}$ For the set-valued version of the IIA-axiom see (Shubik, 1982, p. 421), who attributes it to an informal note of Nash.
} 


$$
(\psi \circ \varphi)(C):=\psi(\varphi(C)) .
$$

Notice that this is indeed a legitimate definition, and that $(\psi \circ \varphi)(C) \subset \varphi(C)$ for all $C \in \mathcal{C}$.

A well-known class of bargaining solutions is the class of (asymmetric) Nash bargaining solutions. For a non-zero vector $\alpha=\left(\alpha_{i}\right)_{i \in N} \in \mathbb{R}^{n}$ the $\alpha$-bargaining solution $N^{\alpha}$ is defined by, for all $C \in \mathcal{C}$,

$$
N^{\alpha}(C)=\left\{x \in C \mid \prod_{i} x_{i}^{\alpha_{i}} \geq \prod_{i} y_{i}^{\alpha_{i}} \text { for all } y \in C\right\} .
$$

A function of the form $x \mapsto \prod_{i} x_{i}^{\alpha_{i}}$ is called a Nash product. A solution $\varphi$ is called a Nash bargaining solution ${ }^{10}$ if there exists a non-zero vector $\alpha \geq 0$ such that $\varphi=N^{\alpha}$. All $\alpha$-bargaining solutions satisfy IIA and COV. If $\alpha \geq 0$, that is, if $N^{\alpha}$ is a Nash bargaining solution, then $N^{\alpha}$ satisfies WPO.

Notice that $N^{\alpha}(C) \in \mathcal{C}$ for any $C \in \mathcal{C}$. Therefore the following definition makes sense. A solution $\varphi$ on $\mathcal{C}$ is an iterated Nash bargaining solution if there are non-zero vectors $\alpha^{1}, \ldots, \alpha^{k} \in \mathbb{R}^{n}$ such that $\alpha^{1} \geq 0$ and $\varphi=N^{\alpha^{k}} \circ \cdots \circ N^{\alpha^{1}}$.

The notions of $\alpha$-bargaining solution, Nash bargaining solution and iterated Nash bargaining solution carry over to solutions on an arbitrary domain $\mathcal{D}$ with $\mathcal{D} \subset \mathcal{C}$ by taking restrictions. Notice that the iterated Nash bargaining solution $N^{\alpha^{k}} \circ \cdots \circ N^{\alpha^{1}}$ is a refinement of the Nash bargaining solution $N^{\alpha^{1}}$. The motivation for the extra requirement $\alpha^{1} \geq 0$ comes from the following observation.

Lemma 2.1 Suppose for a bargaining solution $\varphi: \mathcal{D} \rightarrow \mathbb{R}^{n}$ on domain $\mathcal{D}$ with $\mathcal{F} \subset$ $\mathcal{D} \subset \mathcal{C}$ that $\varphi=N^{\alpha^{k}} \circ \ldots \circ N^{\alpha^{1}}$ for non-zero vectors $\alpha^{1}, \ldots, \alpha^{k} \in \mathbb{R}^{n}$. Then $\varphi$ satisfies WPO if and only if $\alpha^{1} \geq 0$.

Proof Suppose that $\alpha^{1} \geq 0$. Then $N^{\alpha^{1}}$ satisfies WPO. Hence, since $\varphi(C) \subset N^{\alpha^{1}}(C)$ for all $C \in \mathcal{C}$, also $\varphi$ satisfies WPO.

Conversely, suppose w.l.o.g. ${ }^{11}$ that $\alpha_{1}^{1}<0$. Define $x \in \mathbb{R}_{++}^{n}$ by $x_{1}:=2$ and $x_{i}:=1$ for all $i \neq 1$. Clearly $N^{\alpha^{1}}(e, x)=\{e\}$. Then, by the continuity of the Nash product, $N^{\alpha^{1}}(e, x+\varepsilon e)=\{e\}$ for sufficiently small $\varepsilon>0$. Hence, also $\varphi(e, x+\varepsilon e)=\{e\}$ for sufficiently small $\varepsilon>0$. This violates WPO.

Let $\mathcal{D}$ be a domain with $\mathcal{F} \subset \mathcal{D}$. A solution $\varphi: \mathcal{D} \rightarrow \mathbb{R}_{++}^{n}$ is called transitive if, for all $x_{1}, x_{2}, x_{3} \in \mathbb{R}_{++}^{n}$, from $x_{2} \in \varphi\left(x_{1}, x_{2}\right)$ and $x_{3} \in \varphi\left(x_{2}, x_{3}\right)$ it follows that $x_{3} \in \varphi\left(x_{1}, x_{3}\right)$. We shall frequently use the following observation.

Lemma 2.2 Any solution $\varphi: \mathcal{D} \rightarrow \mathbb{R}_{++}^{n}$ that satisfies IIA is transitive.

\footnotetext{
10 These solutions were first introduced-for convex two-person bargaining problems and positive weight vectors-by Harsanyi and Selten (1972).

11 Without loss of generality.
} 
Proof Suppose that $\varphi$ satisfies IIA, that $x_{2} \in \varphi\left(x_{1}, x_{2}\right)$, and that $x_{3} \in \varphi\left(x_{2}, x_{3}\right)$. Suppose that $\varphi\left(x_{1}, x_{2}, x_{3}\right)$ does not contain $x_{3}$. Then, because $x_{3} \in \varphi\left(x_{2}, x_{3}\right)$, it also does not contain $x_{2}$ by IIA. Consequently, because $x_{2} \in \varphi\left(x_{1}, x_{2}\right)$, it does also not contain $x_{1}$ by IIA. This contradicts the assumption that $\varphi\left(x_{1}, x_{2}, x_{3}\right)$ is not empty. Hence, $\varphi\left(x_{1}, x_{2}, x_{3}\right)$ contains $x_{3}$.

In the next section we provide three increasingly detailed characterizations of the class of all WPO, COV, and IIA bargaining solutions on the domain $\mathcal{F}$. In Sect. 4 we show how these characterizations can be further analyzed, depending on which set-theoretic axiom one wishes to adopt. Section 5 presents ensuing existing and new results, and Sect. 6 presents an extension of a theorem by Birkhoff following from our results.

\section{Three characterizations}

\subsection{The first characterization}

On the domain $\mathcal{F}$ we study the set of all WPO, COV and IIA bargaining solutions. In this subsection we show that each WPO, COV and IIA bargaining solution is a refinement of a Nash bargaining solution. Technical proofs can be found in Appendix A.

Let $\varphi: \mathcal{F} \rightarrow \mathbb{R}_{++}^{n}$ be a bargaining solution that satisfies WPO, COV and IIA. Take $x \in \mathbb{R}_{++}^{n}$. Define for $y \in \mathbb{R}_{++}^{n}$,

$$
\lambda(x, y):=\inf \{\lambda>0 \mid \lambda y \in \varphi(x, \lambda y)\} .
$$

By the following lemma this number is well-defined and positive. ${ }^{12}$

Lemma 3.1 Let $x, y \in \mathbb{R}_{++}^{n}$. Then $0<\lambda(x, y)<\infty$.

The next lemma prepares for the definition of the generalized indifference curve below.

Lemma 3.2 For every $x, y \in \mathbb{R}_{++}^{n}$, if $\lambda<\lambda(x, y)$ then $\varphi(x, \lambda y)=\{x\}$, and if $\lambda>\lambda(x, y)$ then $\varphi(x, \lambda y)=\{\lambda y\}$.

Define

$$
I_{\varphi}(x):=\left\{\lambda(x, y) y \mid y \in \mathbb{R}_{++}^{n}\right\} .
$$

The set $I_{\varphi}(x)$ is a subset of $\mathbb{R}_{++}^{n}$. It is called the generalized indifference curve (through $x)$. We briefly discuss the reason for this name. As we will see in Sect. $5, I_{\varphi}(x)$ is indeed the indifference curve through $x$ whenever $\varphi$ satisfies CON, in the sense that $I_{\varphi}(x)$ equals the set of points $y \in \mathbb{R}_{++}^{n}$ with $\varphi(x, y)=\{x, y\}$ in that case.

When $\varphi$ does not (necessarily) satisfy CON, Lemma 3.2 shows that the point $\lambda(x, y) y$ is exactly the point of the form $\lambda y$ on the ray emanating from the origin

\footnotetext{
12 In fact, $\lambda(x, y)$ depends on the solution $\varphi$ and we should write, for example, $\lambda_{\varphi}(x, y)$. Usually, however, it is clear which solution $\varphi$ is meant and we simply write $\lambda(x, y)$.
} 
through $y$ where we switch from choosing $\varphi(x, \lambda y)=\{\lambda y\}$ when $\lambda>\lambda(x, y)$ to choosing $\varphi(x, \lambda y)=\{x\}$ when $\lambda<\lambda(x, y)$. The choice for $\varphi(x, \lambda(x, y) y)$ itself is left unspecified. ${ }^{13}$

We can say this somewhat differently. Notice that $I_{\varphi}(x)=\left\{y \in \mathbb{R}_{++}^{n} \mid \lambda(x, y)=1\right\}$. Thus, $I_{\varphi}(x)$ is the set of points $y \in \mathbb{R}_{++}^{n}$ where we switch from choosing $\varphi(x, \lambda y)=$ $\{\lambda y\}$ when $\lambda>1$ to choosing $\varphi(x, \lambda y)=\{x\}$ when $\lambda<1$. Again, the choice for $\varphi(x, y)$ itself is left unspecified. It can be either $\{x\}$, or $\{y\}$, or $\{x, y\}$ (although this choice cannot be made entirely arbitrarily as we will see in the next subsections).

We show that a WPO, COV and IIA bargaining solution $\varphi$ is in fact a refinement of an $\alpha$-bargaining solution for some non-zero vector $\alpha \geq 0$. In order to specify for exactly which $\alpha$, define $\lambda(x):=\lambda(e, x)$ for $x \in \mathbb{R}_{++}^{n}$. Next, define $a^{j} \in \mathbb{R}_{++}^{n}$ by

$$
a_{i}^{j}:= \begin{cases}\frac{1}{2} & \text { if } i=j \\ 1 & \text { otherwise. }\end{cases}
$$

Define the vector $\alpha=\left(\alpha_{1}, \ldots, \alpha_{n}\right)$ by

$$
\alpha_{j}:={ }^{2} \log \lambda\left(a^{j}\right)
$$

Notice that $e \geq a^{j}$. Hence by WPO, $\lambda\left(a^{j}\right) \geq 1$, and therefore $\alpha_{j} \geq 0$. Moreover, we have the following lemma.

Lemma $3.3 \sum_{i} \alpha_{i}=1$.

Proof We show $\prod_{j} \lambda\left(a^{j}\right)=2$ in two steps.

A. We show that $\lambda(x) \cdot \lambda(y)=\lambda(x y)$ for any $x, y \in \mathbb{R}_{++}^{n}$. Take $x, y \in \mathbb{R}_{++}^{n}$. Take $\lambda>\lambda(x)$ and $\mu>\lambda(y)$. Then $\varphi(\lambda x, e)=\{\lambda x\}$ and $\varphi(\mu y, e)=\{\mu y\}$ by Lemma 3.2. So, by COV and transitivity, $\varphi(\lambda \mu x y, e)=\{\lambda \mu x y\}$ and $\lambda \mu \geq \lambda(x y)$. Hence, $\lambda(x) \cdot \lambda(y) \geq \lambda(x y)$. Conversely, take $\lambda<\lambda(x)$ and $\mu<\lambda(y)$. Then $\varphi(\lambda x, e)=\{e\}$ and $\varphi(\mu y, e)=\{e\}$ by Lemma 3.2. So, by COV and transitivity, $\varphi(\lambda \mu x y, e)=\{e\}$ and $\lambda \mu \leq \lambda(x y)$. Hence, $\lambda(x) \cdot \lambda(y) \leq \lambda(x y)$. Altogether $\lambda(x) \cdot \lambda(y)=\lambda(x y)$.

B. Since $\prod_{j} a^{j}=\frac{1}{2} e$, it is easy to see that $\lambda\left(\prod_{j} a^{j}\right)=2$. Hence, by A, $\prod_{j} \lambda\left(a^{j}\right)=$ $\lambda\left(\prod_{j} a^{j}\right)=2$.

We can now show the first (partial) characterization, namely that $\varphi$ is a refinement of the $\alpha$-Nash bargaining solution, where $\alpha$ is chosen in the above way.

Theorem 3.4 Let $\varphi$ be a WPO, COV and IIA bargaining solution. Then there is a unique non-zero vector $\alpha \geq 0$ such that $\varphi$ is a refinement of the Nash bargaining solution $N^{\alpha}$.

\footnotetext{
13 The now following construction bears similarity to the classical construction of a utility function representing a binary relation (e.g. Debreu 1964). Note, however, that we do not require continuity.
} 
Proof Let $\varphi$ be a bargaining solution that satisfies WPO, COV and IIA. Let $\alpha=$ $\left(\alpha_{1}, \ldots, \alpha_{n}\right)$ be as defined before. Take $F \in \mathcal{F}$ and $x \in F$. Suppose that $x \notin N^{\alpha}(F)$. We show that $x \notin \varphi(F)$.

By IIA it suffices to show that there is a $y \in F$ with $\varphi(x, y)=\{y\}$. Since $x \notin$ $N^{\alpha}(F)$, there is a $y \in F$ with

$$
\prod_{i} x_{i}^{\alpha_{i}}<\prod_{i} y_{i}^{\alpha_{i}}
$$

We show that indeed $\varphi(x, y)=\{y\}$. Write $\mu:=\lambda(x, y)$. Then, by definition, $\mu \cdot y \in$ $I_{\varphi}(x)$. So, by Lemma A.5 and Lemma 3.3,

$$
\prod_{i} x_{i}^{\alpha_{i}}=\prod_{i}\left(\mu \cdot y_{i}\right)^{\alpha_{i}}=\prod_{i} \mu^{\alpha_{i}} \cdot \prod_{i} y_{i}^{\alpha_{i}}=\mu^{\sum_{i} \alpha_{i}} \cdot \prod_{i} y_{i}^{\alpha_{i}}=\mu \cdot \prod_{i} y_{i}^{\alpha_{i}} .
$$

Now from inequality $(*)$ we conclude that $\lambda(x, y)=\mu<1$. Hence, by Lemma 3.2, $\varphi(x, y)=\{y\}$, and the proof is complete.

This shows that the collection of Nash bargaining solutions coincides with the collection of maximal WPO, COV, and IIA bargaining solutions, where maximality is taken with respect to set inclusion. ${ }^{14}$ Conversely, we can view every WPO, IIA and COV bargaining solution as arising from a Nash bargaining solution, with the additional proviso that ties, or indifferences, within the indifference curve of the Nash bargaining solution may be broken one way or the other. How exactly ties can be broken is the topic of the next two subsections.

\subsection{The second characterization}

In this subsection we show that for a WPO, COV and IIA bargaining solution, ties within the indifference curve of the enveloping Nash bargaining solution can only be broken in a specific way, namely only in a way that respects the covariance requirement together with transitivity. We give a precise description of the consequences of this observation.

Let $\varphi: \mathcal{F} \rightarrow \mathbb{R}_{++}^{n}$ be a WPO, COV and IIA bargaining solution. Consider the generalized indifference curve $I_{\varphi}:=I_{\varphi}(e)$ through the unit vector $e$. Notice that $I_{\varphi}$ is an Abelian multiplicative group under the operation defined by $(x y)_{i}:=x_{i} y_{i}$. Indeed, if $x, y \in I_{\varphi}$, then $x y \in I_{\varphi}$ and $\frac{e}{x} \in I_{\varphi}$ by Lemma A.5. Define the subsets $L_{\varphi}, D_{\varphi}$ and $R_{\varphi}$ of $I_{\varphi}$ by

$$
\begin{aligned}
& L_{\varphi}:=\left\{x \in I_{\varphi} \mid \varphi(x, e)=\{x\}, x \neq e\right\} \\
& D_{\varphi}:=\left\{x \in I_{\varphi} \mid \varphi(x, e)=\{x, e\}\right\} \\
& R_{\varphi}:=\left\{x \in I_{\varphi} \mid \varphi(x, e)=\{e\}, x \neq e\right\} .
\end{aligned}
$$

\footnotetext{
14 That is, we can define a partial order on bargaining solutions by $\phi \preceq \psi$ when $\phi(F) \subset \psi(F)$ for all $F \in \mathcal{F}$. The Nash bargaining solutions are the maximal elements w.r.t. this partial order.
} 
Now let $I$ be an Abelian group with respect to the operation *. An LDR decomposition of $I$ is a partition of $I$ into sets $L, D$ and $R$ such that $L, D$ and $R$ are closed under $*, D$ is not empty, $\lambda \in R$ and $\mu \in D$ implies that $\lambda * \mu \in R$, and $\lambda \in L$ and $\mu \in D$ implies that $\lambda * \mu \in L$.

This implies $e \in D$ (where $e$ denotes the neutral element of $I$ ), and if $x \in L$ then $\frac{e}{x} \in R$ (where $\frac{e}{x}$ denotes the inverse of $x$ ). If (in $\mathbb{R}^{k}$ ) the operation $*$ is a multiplication, we say that the LDR decomposition is multiplicative, and we say it is additive if the operation $*$ is an addition.

Example To give the reader a first intuition for these notions, we give two examples of additive LDR decompositions. The first example is $I=\mathbb{R}, D=\{0\}, L=(-\infty, 0)$ and $R=(0, \infty)$. The second example is $I=\mathbb{R}^{2}, D=\{(0,0)\}$,

$$
L=\left\{\left(x_{1}, x_{2}\right) \mid x_{1}>0\right\} \cup\left\{\left(x_{1}, x_{2}\right) \mid x_{1}=0 \text { and } x_{2}>0\right\}
$$

and $R=-L$. It can easily be verified that both examples indeed are LDR decompositions. Note in the second example that sets $L$ and $R$ are neither open nor closed.

Theorem 3.5 The sets $L_{\varphi}, D_{\varphi}$ and $R_{\varphi}$ form an LDR decomposition of the Abelian multiplicative group $I_{\varphi}$.

Proof Clearly $L_{\varphi}, D_{\varphi}$ and $R_{\varphi}$ partition $I_{\varphi}$. Further, since $\varphi$ is transitive and satisfies $\mathrm{COV}, L_{\varphi}, D_{\varphi}$ and $R_{\varphi}$ are closed under multiplication. The set $D_{\varphi}$ is not empty because it contains $e$.

Now take an $x \in R_{\varphi}$ and $y \in D_{\varphi}$. So, using COV, $e \in \varphi(x, e)$ and $x \in \varphi(x y, x)$. Hence, by transitivity, $e \in \varphi(x y, e)$. However, also $x y \notin \varphi(x y, y)$ and $e \in \varphi(y, e)$. Hence, by transitivity, $x y \notin \varphi(x y, e)$. Thus, $\varphi(x y, e)=\{e\}$ and $x y \in R_{\varphi}$.

Similarly it can be shown that $x \in L_{\varphi}$ and $y \in D_{\varphi}$ imply $x y \in L_{\varphi}$. Hence, $L_{\varphi}, D_{\varphi}$ and $R_{\varphi}$ form an LDR decomposition of $I_{\varphi}$.

Thus, each WPO, IIA and COV bargaining solution $\varphi$ determines a quadruple

$$
\left(\alpha, L_{\varphi}, D_{\varphi}, R_{\varphi}\right)
$$

where $\alpha \geq 0$ is such that the set

$$
I_{\alpha}:=\left\{x \in \mathbb{R}_{++}^{n} \mid \prod_{i} x_{i}^{\alpha_{i}}=1\right\}
$$

equals $I_{\varphi}$, and $L_{\varphi}, D_{\varphi}$ and $R_{\varphi}$ constitute an LDR decomposition of $I_{\alpha}$. We call this quadruple the characteristic quadruple of $\varphi$. Let $\mathcal{Q}$ denote the collection of quadruples $(\alpha, L, D, R)$ such that $\alpha=\left(\alpha_{1}, \ldots, \alpha_{n}\right)$ is a non-negative vector with $\sum_{i} \alpha_{i}=1$, and $L, D$ and $R$ form an LDR decomposition of $I_{\alpha} \cdot{ }^{15}$ Let $\mathcal{W}$ denote the collection of WPO, COV and IIA bargaining solutions on $\mathcal{F}$. Let $Q$ denote the map that assigns to each

$\overline{15}$ It is easy to check that $I_{\alpha}$ is an Abelian multiplicative group. 
element $\varphi \in \mathcal{W}$ its characteristic quadruple. Lemma 3.3 together with Theorem 3.5 show that $Q(\varphi) \in \mathcal{Q}$ for each $\varphi \in \mathcal{W}$.

We show now that the characteristic quadruple of $\varphi$ characterizes the solution $\varphi$ in the following sense. Not only does each WPO, IIA and COV bargaining solution determine a (unique) characteristic quadruple in $\mathcal{Q}$, but conversely also each quadruple in $\mathcal{Q}$ uniquely determines a WPO, IIA and COV bargaining solution.

Theorem 3.6 The map $Q: \mathcal{W} \rightarrow \mathcal{Q}$ is one-to-one and onto.

Proof We show that $Q$ has an inverse as follows. Take a quadruple $(\alpha, L, D, R)$ in $\mathcal{Q}$. We show that there exists a unique WPO, COV and IIA bargaining solution $\varphi: \mathcal{F} \rightarrow \mathbb{R}_{++}^{n}$ such that $I_{\varphi}=I_{\alpha}, L_{\varphi}=L, D_{\varphi}=D$ and $R_{\varphi}=R$. by

First take an arbitrary set $\{x, y\} \in \mathcal{F}$. Take $\lambda>0$ such that $\lambda \frac{x}{y} \in I_{\alpha}$. Define $\mu(x, y)$

$$
\mu(x, y):= \begin{cases}\{x\} & \text { if } \lambda<1 \\ \{x\} & \text { if } \lambda=1 \text { and } \frac{x}{y} \in L \\ \{x, y\} & \text { if } \lambda=1 \text { and } \frac{x}{y} \in D \\ \{y\} & \text { if } \lambda=1 \text { and } \frac{x}{y} \in R \\ \{y\} & \text { if } \lambda>1 .\end{cases}
$$

Observe that $\mu(x, y)$ is defined in accordance with what the LDR decomposition $L, D, R$ of $I_{\alpha}$ prescribes for the set $\left\{\frac{x}{y}, e\right\}$. By COV, this also determines how to make the choice for $\{x, y\}$.

We argue that $\mu$ is transitive. Take $x^{1}, x^{2}, x^{3} \in \mathbb{R}_{++}^{n}$. Suppose that $x^{2} \in \mu\left(x^{1}, x^{2}\right)$ and $x^{3} \in \mu\left(x^{2}, x^{3}\right)$. Let $\lambda_{1}>0$ and $\lambda_{2}>0$ be such that $\lambda_{1} \frac{x^{1}}{x^{2}} \in I_{\alpha}$ and $\lambda_{2} \frac{x^{2}}{x^{3}} \in I_{\alpha}$. Then, since $I_{\alpha}$ is a multiplicative group, $\lambda_{1} \lambda_{2} \frac{x^{1}}{x^{3}} \in I_{\alpha}$. Moreover, since $x^{2} \in \mu\left(x^{1}, x^{2}\right)$ and $x^{3} \in \mu\left(x^{2}, x^{3}\right)$, we have that $\lambda_{1} \geq 1$ and $\lambda_{2} \geq 1$. Therefore also $\lambda_{1} \lambda_{2} \geq 1$. We distinguish two cases.

(a) $\lambda_{1} \lambda_{2}>1$. In this case $\mu\left(x^{1}, x^{3}\right)=\left\{x^{3}\right\}$ by definition.

(b) $\quad \lambda_{1} \lambda_{2}=1$. In this case $\lambda_{1}=\lambda_{2}=1$. So, by definition of $\mu, \frac{x^{1}}{x^{2}} \in R \cup D$ and $\frac{x^{2}}{x^{3}} \in R \cup D$. Then, because $L, D$, and $R$ form an LDR decomposition of $I_{\alpha}$, also $\frac{x^{1}}{x^{3}} \in R \cup D$, and hence $x^{3} \in \mu\left(x^{1}, x^{3}\right)$.

Next define $\varphi: \mathcal{F} \rightarrow \mathbb{R}_{++}^{n}$ by, for all $F \in \mathcal{F}$,

$$
\varphi(F):=\{x \in F \mid x \in \mu(x, y) \text { for all } y \in F\} .
$$

We show that $\varphi$ is the unique WPO, IIA and COV bargaining solution $\varphi: \mathcal{F} \rightarrow \mathbb{R}_{++}^{n}$ such that $\varphi(x, y)=\mu(x, y)$ for all $x, y \in \mathbb{R}_{++}^{n}$.

A. From the definition of $\varphi$ it follows immediately that $\varphi(x, y)=\mu(x, y)$ for all $x, y \in \mathbb{R}_{++}^{n}$. From this it follows that $I_{\varphi}=I_{\alpha}, L_{\varphi}=L, D_{\varphi}=D$ and $R_{\varphi}=R$ (once we established that $\varphi$ is a bargaining solution and that it satisfies WPO, IIA, and COV), since all these sets are defined by considering pairs of elements in $\mathbb{R}_{++}^{n}$. 
B. Take $F \in \mathcal{F}$. We claim that $\varphi(F)$ is not empty. Suppose it were empty. Take a point $x_{1} \in F$. Since $x_{1} \notin \varphi(F)$ there is an $x_{2} \in F$ with $x_{1} \notin \mu\left(x_{1}, x_{2}\right)$. Since $\mu\left(x_{1}, x_{2}\right)$ is not empty, necessarily $\left\{x_{2}\right\}=\mu\left(x_{1}, x_{2}\right)$. In the same way we find an $x_{3} \in F$ with $x_{2} \notin \mu\left(x_{2}, x_{3}\right)$. Iterating this procedure yields a sequence $x_{1}, x_{2}, x_{3}, \ldots$ in $F$ with $x_{k} \notin \mu\left(x_{k}, x_{k+1}\right)$ for all $k \geq 1$. Since $F$ is finite, we have $x_{r}=x_{t}$ for some $r, t$ with $r<t$. This violates the transitivity of $\mu$.

C. The solution $\varphi$ satisfies IIA. Take $F, G \in \mathcal{F}$ with $G \subset F$ and $G \cap \varphi(F) \neq \phi$. We show that $\varphi(G)=G \cap \varphi(F)$.

C1. Take an $x \in \varphi(G)$. Clearly $x \in G$. In order to show that $x \in \varphi(F)$, take $y \in F$. If $x \in \mu(x, y)$ we are done. Since $G \cap \varphi(F) \neq \phi$, we can take $z \in G \cap \varphi(F)$. Since $x \in \mu(x, z)$ and $z \in \mu(y, z)$, we have $x \in \mu(x, y)$ by the transitivity of $\mu$.

C2. Take an $x \in G \cap \varphi(F)$. Take a $y \in G$. Then $x \in \mu(x, y)$ since $x \in \varphi(F)$. Hence $x \in \varphi(G)$.

D. Next we show that $\varphi$ satisfies WPO. Take $F \in \mathcal{F}$ and $x, y \in F$ with $x<y$. Then $\frac{x}{y}<e$. So, for $\lambda>0$ with $\lambda \frac{x}{y} \in I_{\alpha}$ we have $\lambda>1$. Hence, $\mu(x, y)=\{y\}$ and therefore $x \notin \varphi(F)$ by the definition of $\varphi$.

E. We show that $\varphi$ satisfies COV. Take $a \in \mathbb{R}_{++}^{n}$, take $F \in \mathcal{F}$ and take $x \in \varphi(F)$. It suffices to show that $a x \in \varphi(a F)$. Take an arbitrary $y \in a F$. Then $\frac{y}{a} \in F$. So, $x \in \mu\left(x, \frac{y}{a}\right)$. Hence, $a x \in \mu(a x, y)$ by the definition of $\mu$.

F. Finally we prove uniqueness. Let $\psi$ be an arbitrary WPO, IIA and COV bargaining solution such that $\psi(x, y)=\mu(x, y)$ for all $x, y \in \mathbb{R}_{++}^{n}$. Take $F \in \mathcal{F}$. We prove that $\psi(F)=\{x \in F \mid x \in \mu(x, y)$ for all $y \in F\}$.

F1. Take an $x \in \psi(F)$. Take a $y \in F$. Then, from IIA and the fact that $\psi(x, y)=$ $\mu(x, y)$, it follows that $x \in \mu(x, y)$.

F2. Take an $x \in F$ with $x \in \mu(x, y)$ for all $y \in F$. Since $\psi(F)$ is not empty, we can take $z \in \psi(F)$. Then $x \in \mu(x, z)=\psi(x, z)$, and hence $x \in \psi(F)$ by IIA.

\subsection{The third characterization}

In this subsection we further refine the characterization in Theorem 3.6 by taking the analysis of the internal structure of the LDR decomposition a few steps further.

Let $\varphi: \mathcal{F} \rightarrow \mathbb{R}_{++}^{n}$ be a WPO, COV and IIA bargaining solution. We use a recursive process to construct a sequence $I_{\varphi}=J_{1} \supset J_{2} \supset \ldots$ of closed multiplicative subspaces $J_{k}$ of $I_{\varphi}$ such that $D:=D_{\varphi} \subset J_{k}$ for all $k$.

STEP I. This is the initialization step. Take $J_{1}:=I_{\varphi}$. Go to STEP II.

STEP II. This is the recursion step. Suppose $J_{k}$ has already been defined. Define $L_{k}:=L_{\varphi} \cap J_{k}$ and $R_{k}:=R_{\varphi} \cap J_{k}$. If $L_{k}$ is empty, define $J_{k+1}:=J_{k}$. If $L_{k}$ is not empty, define $J_{k+1}:=\bar{L}_{k} \cap \bar{R}_{k}$. (Recall that, for a set $S, \bar{S}$ indicates the closure of $S$.) Go to STEP II. ${ }^{16}$

\footnotetext{
16 Of course, we can also formulate this step using the sets $R_{k}$ as criterion, since in an arbitrary LDR decomposition the set $R$ consists exactly of the inverses of the elements of $L$. Similarly, Lemma 3.7 also holds with $R_{\varphi}$ in the place of $L_{\varphi}$. Similar remarks hold for Theorems 3.9 and 3.10.
} 
Note that $J_{1}$ is closed by Lemma A.2. Then an induction argument shows that for all $k, J_{k}$ is closed and $J_{k} \supset J_{k+1}$. Thus we have a sequence $I_{\varphi}=J_{1} \supset J_{2} \supset \cdots$ of closed sets. Further, since $L_{k}, D$, and $R_{k}$ form an LDR decomposition of $J_{k}$ (in particular, $D \subset J_{k}$ by Theorem B.1), it follows from Theorem B.1 that $J_{k+1}$ is a multiplicative subspace of $J_{k}$ with $D \subset J_{k+1}$. Thus, $D$ is a subset of $J_{k}$ for all $k$. Furthermore, if $n_{k}$ denotes the dimension of the closed subspace $J_{k}$, it follows from Theorem B.1 that $n_{k+1}$ is either equal to $n_{k}$ or equal to $n_{k}-1$. Thus the sequence $I=J_{1} \supset J_{2} \supset \ldots$ consists of an initial part $J_{1} \supset \ldots \supset J_{K}$ for which $n_{k+1}=n_{k}-1$ holds for all $k<K$, after which we have that $J_{k}=J_{K}$ for all $k \geq K$.

The (uniquely defined) number $K$ is called the characteristic number of the LDR decomposition $L_{\varphi}, D_{\varphi}$ and $R_{\varphi}$ of $I_{\varphi}$. The sequence $J_{1} \supset \ldots \supset J_{K}$ is called the central chain of the LDR decomposition. The linear subspace $J_{\varphi}:=J_{K}$ of $I_{\varphi}$ is called the center of the LDR decomposition. Evidently $D_{\varphi} \subset J_{\varphi}$.

Lemma 3.7 Let $J_{\varphi}$ be the center of the $\mathrm{LDR}$ decomposition $L_{\varphi}, D_{\varphi}$ and $R_{\varphi}$ of $I_{\varphi}$. Then either $L_{\varphi} \cap J_{\varphi}$ is empty or $L_{\varphi} \cap J_{\varphi}$ is dense in $J_{\varphi}$.

Proof Suppose that $L_{\varphi} \cap J_{\varphi}$ is not empty. Let $K$ be the characteristic number of the LDR decomposition $L_{\varphi}, D_{\varphi}$ and $R_{\varphi}$ of $I_{\varphi}$. Then $J_{K+1}:=\overline{L_{\varphi} \cap J_{K}} \cap \overline{R_{\varphi} \cap J_{K}}$ is equal to $J_{K}$. Hence, $L_{\varphi} \cap J_{\varphi}=L_{\varphi} \cap J_{K}$ is dense in $J_{K}=J_{\varphi}$.

\subsubsection{Canonical coefficients}

Let $\varphi: \mathcal{F} \rightarrow \mathbb{R}_{++}^{n}$ be a WPO, COV and IIA bargaining solution. Let $\alpha$ be as constructed in Sect. 3.1. Let $K$ be the characteristic number of the LDR decomposition $L_{\varphi}, D_{\varphi}$, and $R_{\varphi}$ of $I_{\varphi}=I_{\alpha}$, and let $I_{\varphi}=J_{1} \supset \cdots \supset J_{K}=J_{\varphi}$ be the central chain.

We construct a sequence of orthogonal non-zero vectors $\beta^{1}, \ldots, \beta^{K}$ in $\mathbb{R}^{n}$ such that $\beta^{1}=\alpha$, and for all $2 \leq k \leq K$,

$$
J_{k}=\left\{x \in J_{k-1} \mid \prod_{j} x_{j}^{\beta_{j}^{k}}=1\right\} .
$$

Evidently, we take $\beta^{1}:=\alpha$. We recursively define $\beta^{2}, \ldots, \beta^{K}$ as follows. Suppose that, for $2 \leq k \leq K, \beta^{k-1}$ has already been defined. Write $H_{k-1}:=\left\{y \in \mathbb{R}^{n} \mid\right.$ $\left.\sum_{i} \beta_{i}^{k-1} y_{i}=0\right\}$.

Lemma 3.8 There exists a vector $\beta^{k} \in H_{k-1}$ such that

$$
J_{k}=\left\{x \in J_{k-1} \mid \prod_{i} x_{i}^{\beta_{i}^{k}}=1\right\} .
$$

This vector is unique up to scalar multiplication.

Proof Define the operation $\rho: J_{1} \rightarrow \mathbb{R}^{n}$ by

$$
\rho\left(x_{1}, \ldots, x_{n}\right):=\left(\ln \left(x_{1}\right), \ldots, \ln \left(x_{n}\right)\right) .
$$


Since $J_{1}=I_{\phi}=I_{\alpha}=I_{\beta}$,

$$
J_{1}=\left\{x \in \mathbb{R}_{++}^{N} \mid \prod_{i} x_{i}^{\beta_{i}^{1}}=1\right\} .
$$

Then it is straightforward to check that $\rho\left(J_{1}\right)=H_{1}$, that $\rho$ is a homeomorphism from $J_{1}$ to $H_{1}$, and that $\rho$ is an isomorphism w.r.t. addition on $H_{\alpha}$ and multiplication on $I_{\alpha}$.

Now it is easy to check that $\rho\left(J_{k-1}\right)=H_{k-1}$. So, since $J_{k}$ is a closed multiplicative subspace of $J_{k-1}$, it follows that $\rho\left(J_{k}\right)$ is a closed additive subspace of $H_{k-1}$. Moreover, $\operatorname{dim}\left(J_{k}\right)=\operatorname{dim}\left(J_{k-1}\right)-1$ by construction. Hence, $\rho\left(J_{k}\right)$ is a linear subspace of $H_{k-1}$ of dimension $\operatorname{dim}\left(H_{k-1}\right)-1$. So there exists a vector $\beta^{k} \in H_{k-1}$ (which is unique up to scalar multiplication) such that

$$
\rho\left(J_{k}\right):=\left\{y \in H_{k-1} \mid \sum_{i} \beta_{i}^{k} y_{i}=0\right\} .
$$

Using the isomorphism $\rho$ it is now easy to show that $J_{k}=\left\{x \in J_{k-1} \mid \prod_{i} x_{i}^{\beta_{i}^{k}}=1\right\}$.

Moreover, by taking $-\beta^{k}$ instead of $\beta^{k}$ when necessary, we can use Lemma B.2 to guarantee that

$$
\left\{x \in J_{k-1} \mid \prod_{j} x_{j}^{\beta_{j}^{k}}>1\right\} \subset L_{\varphi} \cap J_{k-1} .
$$

The vectors $\beta^{1}, \ldots, \beta^{K}$ are called the canonical coefficients of $\varphi$. Each of these vectors is unique up to multiplication by a positive scalar. Note that, because $\beta^{k} \in H_{k-1}$ for all $2 \leq k \leq K$, the vectors $\beta^{k}$ are orthogonal.

\subsubsection{The characterization}

Theorem 3.9 Let $\varphi: \mathcal{F} \rightarrow \mathbb{R}_{++}^{n}$ be a WPO, COV, and IIA bargaining solution. Let $\beta^{1}, \ldots, \beta^{K}$ be the canonical coefficients of $\varphi$. Write $N=N^{\beta^{k}} \circ \cdots \circ N^{\beta^{1}}$. Then either $\varphi=N$ or $\varphi$ is a (strict) refinement of $N$ and $R_{\varphi} \cap D_{N}$ is dense in $D_{N}$.

Proof The proof is in two parts.

A. We show that $\varphi$ is a refinement of $N$. Note that $I_{N}=I_{\beta^{1}}=I_{\alpha}=I_{\varphi}$. Therefore, by Theorem 3.6, it suffices to prove that $L_{N} \subset L_{\varphi}$. Take $x \in L_{N}$. Then $x \in I_{N}$, but $x \notin D_{N}=J_{\varphi}$. Hence, there is a (uniquely determined) $2 \leq k \leq K$ such that $x \in J_{k-1}$, but $x \notin J_{k}$. Thus, because $J_{k}=\left\{x \in J_{k-1} \mid \prod_{i} x_{i}^{\beta_{i}^{k}}=1\right\}$ and $x \in L_{N}$, it follows that $\prod_{j} x_{j}^{\beta_{j}^{k}}>1$. However, by the choice of the canonical coefficients, $\left\{x \in J_{k-1} \mid \prod_{j} x_{j}^{\beta_{j}^{k}}>1\right\} \subset L_{\varphi} \cap J_{k-1}$, and $x \in L_{\varphi}$. 
B. Suppose that $\varphi$ does not equal $N$. We show that $R_{\varphi} \cap D_{N}$ is dense in $D_{N}$. Since $D_{N}=J_{\varphi}$, it follows from Lemma 3.7, with $R_{\varphi}$ in the role of $L_{\varphi}$, that $R_{\varphi} \cap$ $D_{N}$ is either empty or dense in $D_{N}$. Suppose it is empty. Then $D_{\varphi}=J_{\varphi}=$ $D_{N}$, and $\varphi=N$. This contradicts our assumption. Hence $R_{\varphi} \cap D_{N}$ is dense in $D_{N}$.

Theorem 3.10 Let $N: \mathcal{F} \rightarrow \mathbb{R}_{++}^{n}$ be an iterated Nash bargaining solution. Further, let $L, D$ and $R$ be an $L D R$ decomposition of $I:=D_{N}$ such that $R$ is dense in $I$. Then there is a (unique) WPO, COV, and IIA refinement $\varphi$ of $N$ such that $R_{\varphi} \cap I=R$.

Proof Let $\beta^{1}, \ldots, \beta^{K}$ be the canonical coefficients of $N$. Let $\varphi$ be an arbitrary WPO, COV and IIA refinement of $N$, and let $\left(\alpha, L_{\varphi}, D_{\varphi}, R_{\varphi}\right)$ be its characteristic quadruple. Then necessarily $\alpha=\beta^{1}$. Moreover, necessarily $R_{\varphi} \supset R_{N}$. This however implies that $R_{\varphi}=R_{N} \cup R$. Hence, using Theorem 3.6, The bargaining solution that is associated with the characteristic quadruple $\left(\beta^{1}, L_{N} \cup L, D, R_{N} \cup R\right)$ is the unique WPO, COV, and IIA refinement of $N$ such that $R_{\varphi} \cap I=R$.

Thus, every bargaining solution $\varphi: \mathcal{F} \rightarrow \mathbb{R}_{++}^{n}$ is uniquely characterized by an iterated Nash bargaining solution $N$ plus, unless $\varphi=N$, an LDR decomposition $L, D$, and $R$ of $D_{N}$ for which $R$ is a dense subset of $D_{N}$.

The remainder of this paper is devoted to discussing known and new consequences of the characterizations derived in this section.

\section{To be or not to be}

In this section we use the characterizations derived in the preceding section to discuss the existence of WPO, COV and IIA bargaining solutions that are not iterated Nash bargaining solutions. We divide the collection of WPO, COV and IIA bargaining solutions into two types: iterated Nash bargaining solutions are of type I and all other WPO, COV and IIA bargaining solutions are of type II.

On some domains, solutions of the second type do not exist, see e.g. Naumova and Yanovskaya (2001) and Theorem 5.4 below. Furthermore, examples of WPO, COV and IIA bargaining solutions other than iterated Nash bargaining solutions have, to the best of our knowledge, never been reported in literature. The question arises whether bargaining solutions of the second type exist at all on the domain $\mathcal{F}$, or on any larger domain. The answer to this question is ambiguous: it depends!

If we accept (for example) the Axiom of Choice, it is possible to construct (many) WPO, COV and IIA bargaining solutions on the domain $\mathcal{F}$ that are not iterated Nash bargaining solutions. However, in mathematical logic there is an alternative for the Axiom of Choice, called the Axiom of Determinacy. The Axiom of Determinacy is compatible with the countable version of the Axiom of Choice, but it is not compatible with the uncountable version of the Axiom of Choice. If we accept the Axiom of Determinacy, bargaining solutions of the second type do not exist. In this section we explore both avenues and provide proofs for these claims. 


\subsection{Not to be: the Axiom of Determinacy}

In this subsection we show that, when we accept the Axiom of Determinacy, WPO, COV and IIA bargaining solutions of type II do not exist on the domain $\mathcal{F}$. Hence, in this case, on the domain $\mathcal{F}$, and therefore on any other domain $\mathcal{D}$ with $\mathcal{F} \subset \mathcal{D} \subset \mathcal{C}$, the collection of all WPO, COV and IIA bargaining solutions coincides with the collection of all iterated Nash bargaining solutions.

THE AXIOM OF DETERMINACY. Consider a subset $A$ of the unit interval $[0,1]$. The set $A$ defines a game $G(A)$ for two players, I and II. This game is played as follows. First player I chooses a binary number $a_{1} \in\{0,1\}$. Next, player II observes this number and subsequently chooses a binary number $a_{2} \in\{0,1\}$. Then Player I observes what Player II chose, and chooses a third binary number $a_{3} \in\{0,1\}$. Etcetera. This way Players I and II construct an infinite sequence $a_{1}, a_{2}, a_{3}, a_{4}, \ldots$ of binary numbers, which defines a real number

$$
x=\sum_{k=1}^{\infty} a_{k}\left(\frac{1}{2}\right)^{k}
$$

in the unit interval $[0,1]$. If $x \in A$, Player I wins and if $x \notin A$ Player II wins.

The set $A$ is said to be determined if either Player I or Player II has a winning strategy in the game $G(A)$. The Axiom of Determinacy states that every subset of the unit interval $[0,1]$ is determined. Mycielski and Swierczkowski (1964) showed the following fact.

Theorem 4.1 Every determined set $A$ in $[0,1]$ is measurable.

WPO, COV AND IIA BARGAINING SOLUtions. Thus, the Axiom of Determinacy effectively rules out the existence of sets that are not measurable. We exploit this fact in the remainder of this section to prove that every WPO, COV and IIA bargaining solution on the domain $\mathcal{F}$ is an iterated Nash bargaining solution.

Lemma 4.2 Let $L, D$ and $R$ be an additive LDR decomposition of $\mathbb{R}$. Suppose that $R$ is dense in $\mathbb{R}$. Then $R$ is not a measurable set.

Proof Suppose that $R$ is a measurable set. Let $\lambda$ denote the Borel-Lebesgue measure on $\mathbb{R}$. We derive a contradiction in three steps.

STEP I. Take $u, v \in \mathbb{R}, u<v$. We show that $\lambda(R \cap(u, v)) \leq \frac{1}{2}(v-u)$. Suppose that $\lambda(R \cap(u, v))>\frac{1}{2}(v-u)$. Since $R$ is dense in $\mathbb{R}$ and $L=-R$, also $L$ is dense in $\mathbb{R}$. Therefore we can take $k, l \in L$ with $k<l$ such that $\lambda(R \cap(k, l))>\frac{1}{2}(l-k)$. Now notice that, since $L=-R$ and $R$ is measurable, it follows that

$$
-(R \cap(k, l))=-R \cap(-l,-k)=L \cap(-l,-k)
$$

is a measurable subset of $L$. Therefore, since $k, l \in L$ and $L+L \subset L$, it follows that $(k+l)+(L \cap(-l,-k))$ is a measurable subset of the (measurable) set $L \cap(k, l)$. 
Hence, due to the translation invariance and rotation invariance of the Borel-Lebesgue measure $\lambda$,

$$
\begin{aligned}
\lambda(L \cap(k, l)) & \geq \lambda((k+l)+(L \cap(-l,-k)))=\lambda(L \cap(-l,-k)) \\
& =\lambda(-(R \cap(k, l)))=\lambda(R \cap(k, l)) \\
& >\frac{1}{2}(l-k) .
\end{aligned}
$$

This however contradicts the assumption that $R$ and $L$ are disjoint.

STEP II. Take $u, v \in \mathbb{R}, u<v$. We show that $A:=R \cap(u, v)$ is negligible. Take $\varepsilon>0$. Since $A$ is a measurable set, Theorem 1.1 in Billingsley (1968) implies that there exists an open set $U \supset A$ such that $\lambda(U \backslash A)<\varepsilon$. Since $U$ is an open set in $\mathbb{R}$, we can write $U$ as the disjoint union of open intervals $\left(u_{n}, v_{n}\right), n \in \mathbb{N}$. Hence, using step I to obtain the second inequality,

$$
\begin{aligned}
\sum_{n}\left(v_{n}-u_{n}\right)=\lambda(U) & \leq \lambda(A)+\varepsilon \\
& =\lambda(A \cap U)+\varepsilon \\
& =\sum_{n}^{n} \lambda\left(A \cap\left(u_{n}, v_{n}\right)\right)+\varepsilon \\
& =\sum_{n}^{n} \lambda\left(R \cap\left(u_{n}, v_{n}\right)\right)+\varepsilon \\
& \leq \frac{1}{2} \cdot \sum_{n}\left(v_{n}-u_{n}\right)+\varepsilon .
\end{aligned}
$$

Thus $\lambda(U)=\sum_{n}\left(v_{n}-u_{n}\right) \leq 2 \varepsilon$. This implies that $A$ is negligible.

STEP III. By step II we know that $R$ and $L$ are negligible. Hence, since $L, D$ and $R$ partition $\mathbb{R}, D$ is measurable and $\lambda(D)>0$. However, since $R$ is dense in $\mathbb{R}$, we can take an $r \in R$. Then $r+D$ is a subset of $R$ and $\lambda(r+D)>0$ by the translation invariance of the Borel-Lebesgue measure. This contradicts the negligibility of $R$.

Lemma 4.3 Let $H$ be a linear subspace of $\mathbb{R}^{n}$. Further, let $L D$ and $R$ be an (additive) LDR decomposition of $H$ and suppose that $R$ is dense in $H$. Then there exists a line $P$ in $H$ through the origin such that $P \cap R$ is dense in $P$.

Proof Let $z_{1}, \ldots, z_{k}$ be a basis for $H$. We say that a basis element $z_{i}$ is superfluous when at least one of the following two inclusions holds:

(1) $\left\{\lambda z_{i} \mid \lambda \geq 0\right\} \subset L \cup D$

(2) $\left\{\lambda z_{i} \mid \lambda \leq 0\right\} \subset L \cup D$.

We argue by contradiction that not all basis elements are superfluous. Suppose that all basis elements are superfluous. Assume w.l.o.g. that (1) holds for all $i$ (when only (2) holds, take $-z_{i}$ instead of $z_{i}$ ). Then the set $G$ of points $z \in H$ for which there are non-negative numbers $\lambda_{1}, \ldots, \lambda_{k}$ such that $z=\sum_{i} \lambda_{i} z_{i}$ is a subset of $L \cup D$ which is of full dimension in $H$. This contradicts the density of $R$ in $H$.

Now take a basis element $z_{i}$ that is not superfluous. Then there are $\lambda>0$ and $\mu<0$ such that both $\lambda z_{i}$ and $\mu z_{i}$ are elements of $R$. The result now follows from the fact that $R$ is closed under addition. 
Theorem 4.4 Assume the Axiom of Determinacy. Then a bargaining solution $\varphi: \mathcal{F} \rightarrow$ $\mathbb{R}_{++}^{n}$ satisfies WPO, COV, and IIA if and only if it is an iterated Nash bargaining solution.

Proof Let $\varphi: \mathcal{F} \rightarrow \mathbb{R}_{++}^{n}$ be a WPO, COV, and IIA bargaining solution, and assume that it is not an iterated Nash bargaining solution. Then, by Theorem 3.9 there exists a (unique) iterated Nash bargaining solution $N$ such that $\varphi$ is a strict refinement of $N$ and $R_{\varphi} \cap D_{N}$ is dense in $D_{N}$.

Define the operation $\rho: D_{N} \rightarrow \mathbb{R}^{n}$ by

$$
\rho\left(x_{1}, \ldots, x_{n}\right):=\left(\ln \left(x_{1}\right), \ldots, \ln \left(x_{n}\right)\right)
$$

Write $H:=\rho\left(D_{N}\right)$. It is straightforward to check that $H$ is a closed and additive, and hence linear, subspace of $\mathbb{R}^{n}$. Furthermore, $L:=\rho\left(L_{\varphi} \cap D_{N}\right), D:=\rho\left(D_{\varphi}\right)$ and $R:=\rho\left(R_{\varphi} \cap D_{N}\right)$ form an LDR decomposition of $H$, and $R$ is dense in $H$. So, by Lemma 4.3 there is a line $P$ in $H$ through the origin such that $P \cap R$ is dense in $P$. The existence of such a line is contradicted by Theorem 4.1 and Lemma 4.2.

In fact Lemmas 4.3 and 4.2 show that the above result can also be obtained under the assumption that all sets are measurable, a much weaker assumption than the Axiom of Determinacy.

\subsection{To be: Zorn's Lemma and the Axiom of Choice}

Thus, when we assume the Axiom of Determinacy, the collection of all WPO, COV, and IIA bargaining solutions on $\mathcal{F}$, or any other domain $\mathcal{D}$ with $\mathcal{F} \subset \mathcal{D} \subset \mathcal{C}$, coincides with the collection of all iterated Nash bargaining solutions on that domain. Without the Axiom of Determinacy however, the picture changes. If, instead, we assume Zorn's Lemma, a statement that is equivalent to the Axiom of Choice, it is possible to construct WPO, COV, and IIA bargaining solutions that are not iterated Nash bargaining solutions. We show how this can be done. Let $H_{\alpha} \subset \mathbb{R}^{n}$ be defined by

$$
H_{\alpha}:=\left\{x \in \mathbb{R}^{n} \mid \sum_{i} \alpha_{i} x_{i}=0\right\}
$$

Any additive functional $f: H_{\alpha} \rightarrow \mathbb{R}$ defines an LDR decomposition as follows. Note that the operation $\rho: I_{\alpha} \rightarrow H_{\alpha}$ defined by

$$
\rho\left(x_{1}, \ldots, x_{n}\right):=\left(\ln \left(x_{1}\right), \ldots, \ln \left(x_{n}\right)\right)
$$

is an isomorphism w.r.t. addition on $H_{\alpha}$ and multiplication on $I_{\alpha}$. Define

$$
\begin{aligned}
& L_{f}:=\left\{x \in I_{\alpha} \mid(f \circ \rho)(x)<0\right\} \\
& D_{f}:=\left\{x \in I_{\alpha} \mid(f \circ \rho)(x)=0\right\} \\
& R_{f}:=\left\{x \in I_{\alpha} \mid(f \circ \rho)(x)>0\right\} .
\end{aligned}
$$


It is easy to check that $L_{f}, D_{f}$ and $R_{f}$ form an LDR decomposition of $I_{\alpha}$. Thus, every additive functional $f$ induces a WPO, IIA and COV bargaining solution $\varphi(f)$, and two such functionals $f$ and $g$ induce different solutions $\varphi(f)$ and $\varphi(g)$ whenever $L_{f}, D_{f}, R_{f}$ is a different LDR decomposition than $L_{g}, D_{g}, R_{g}$.

We use the above observation to construct a plethora of WPO, IIA and COV bargaining solutions as follows. Of course every linear functional $f: H_{\alpha} \rightarrow \mathbb{R}$ induces a solution $\varphi(f)$. However, using Zorn's Lemma, we can construct many more different additive functionals, and hence many more different WPO, IIA and COV bargaining solutions. First we need some notation. Let $X$ be a non-empty set and let $\preceq$ be a binary relation on $X$ that satisfies

[1] (reflexivity) for all $x \in X$ we have $x \preceq x$, and

[2] (transitivity) for all $x, y, z \in X$ we have $x \preceq z$ whenever both $x \preceq y$ and $y \preceq z$.

We say that $\preceq$ is a partial order on $X$. A subset $C$ of $X$ is called a chain if for any two elements $x$ and $y$ of $C$ we have at least one of the two inequalities $x \preceq y$ and $y \preceq x$. A chain $C$ of $X$ is said to have an upper bound if there exists an element $a$ of $X$ such that $x \preceq a$ for all $x$ in $C$. An element $a$ of $X$ is called maximal if for any $x$ in $X$ the inequality $a \preceq x$ only holds if $x \preceq a$ holds as well.

Lemma 4.5 (Zorn) Suppose that every chain of X has an upper bound. Then $X$ has a maximal element.

Using Zorn's Lemma we can easily prove the following result, due to Hamel (1905).

Theorem 4.6 $\mathbb{R}^{n}$ has a basis over $\mathbb{Q}$. Consequently, also $H_{\alpha}$ has a basis over $\mathbb{Q}$.

Proof Let $X$ be the set of $\mathbb{Q}$-independent sets in $\mathbb{R}^{n}$, ordered by set inclusion. Let $C$ be a chain in $X$. Then $\cup_{B \in C} B$ is an upper bound of $C$. Hence, by Zorn's Lemma, $X$ has a maximal element, say $B$. It is straightforward to prove that $B$ is a basis of $\mathbb{R}^{n}$ over $\mathbb{Q}$. The second part of the theorem follows from the observation that $H_{\alpha}$ is linearly isomorphic to $\mathbb{R}^{n-1}$.

We construct a multitude of WPO, IIA and COV bargaining solutions as follows. Let $B$ be a basis of $H_{\alpha}$ over $\mathbb{Q}$. An assignment for $B$ is a function $f: B \rightarrow \mathbb{R}$. It is clear that every assignment $f: B \rightarrow \mathbb{R}$ uniquely extends to an additive functional $f: H_{\alpha} \rightarrow \mathbb{R} .^{17}$ Thus, there are as many additive functionals on $H_{\alpha}$ as there are assignments on $B$. We already observed that each additive functional induces a WPO, IIA and COV bargaining solution. Hence, we have the following theorem.

Theorem 4.7 For every assignment $f$ for $B$ there exists a unique WPO, IIA and COV bargaining solution $\varphi(f)$ such that $L_{f}=L_{\varphi(f)}, D_{f}=D_{\varphi(f)}$ and $R_{f}=R_{\varphi(f)}$. Consequently, for any two assignments $f$ and $g, \varphi(f) \neq \varphi(g)$ exactly if $\left(L_{f}, D_{f}, R_{f}\right) \neq$ $\left(L_{g}, D_{g}, R_{g}\right)$.

\footnotetext{
17 Formally we should use a different symbol to indicate the additive function, but we allow for a slight abuse of notation here.
} 
Note that $\varphi(f)$ might still be equal to $\varphi(g)$, even when $f \neq g$ (for example, when $g=2 f)$. Nevertheless, the qualification 'plethora' still applies for the following reason.

Theorem 4.8 Assume the Axiom of Choice. Then there exists a WPO, COV and IIA bargaining solution on $\mathcal{F}$ that is of type II. The cardinality of the collection of type II bargaining solutions is strictly larger than the cardinality of the collection of iterated Nash bargaining solutions.

Proof Notice that $B$ is uncountable. Further, $\left(L_{f}, D_{f}, R_{f}\right)$ is a different LDR decomposition of $I_{\alpha}$ than $\left(L_{g}, D_{g}, R_{g}\right)$ at least when there is a $b \in B$ for which for example $f(b)>0$ and $g(b) \leq 0$ (because in that case $\rho^{-1}(b) \in R_{f}$ while $\rho^{-1}(b)$ $\left.\notin R_{g}\right)$.

Thus the number of different LDR decompositions that can be constructed this way is still at least as large as the cardinality of $3^{B}$. On the other hand the set of all iterated Nash bargaining solutions clearly has the cardinality of $\mathbb{R}^{n}$. Now, $B$ necessarily has the cardinality of $\mathbb{R}^{n}$, while, by the Cantor argument, $3^{B}$ has a higher cardinality than $B$. In particular, not every solution generated in the above way is an iterated Nash bargaining solution, and type II bargaining solutions must necessarily exist.

\subsection{Not to be redux: definability}

The fact that type II WPO COV and IIA bargaining solutions involve non-measurable sets is an interesting observation in itself, irrespective of the question whether one accepts or rejects the Axiom of Determinacy. It shows that the "construction" of type II bargaining solutions necessarily involves the construction of non-measurable sets. It is not clear though what such a "construction" should entail, because nonmeasurable sets can in a very real sense not be constructed using only elementary construction rules. ${ }^{18}$ In this section we show that this observation can be made into a formal statement. For notation we borrow heavily from Zame (2007) and refer to that paper for an excellent discussion of the relevant recent advancements in axiomatic set theory.

A set $A \subset \mathbb{R}^{n}$ is definable if there exists a set-theoretic formula $\Phi\left(t, r, \alpha_{1}, \alpha_{2}, \ldots\right)$ in which $r$ is a real number, $\alpha_{1}, \alpha_{2}, \ldots$ is a sequence of ordinal numbers, and $t$ is the only free variable, such that

$$
A=\left\{x \in \mathbb{R}^{n} \mid \Phi\left(x, r, \alpha_{1}, \alpha_{2}, \ldots\right)\right\}
$$

\footnotetext{
18 An interesting observation in this context (pointed out to us by Professor Jan Mycielski) is that, when we assume ZFC plus the existence of a large cardinal, the subclass $L[R]$ of the universe $V$ of all sets forms a model of $\mathrm{ZF}$ in which $\mathrm{AD}$ as well as a version of $\mathrm{AC}$ known as the Axiom of Dependent Choice (DC) are true. DC still allows one to perform virtually all constructions known within analysis. A comprehensive discussion can be found in Marek and Mycielski (2001).
} 
In Zame (2007) it is explained that the proposition "there exists a definable set that is not Lebesgue measurable" is not provable from the Zermelo-Fraenkel Axioms together with the Axiom of Choice. To flip this statement around, when we can prove in ZFC that a set $A$ is not measurable, then we cannot prove in ZFC that $A$ is a definable set.

This enables us to prove the following statement. We assume the ZF Axioms, together with AC. So, indeed type II WPO, COV and IIA bargaining solutions exist on domain $\mathcal{F}$. For these we can show the following.

Theorem 4.9 Let $\varphi: \mathcal{F} \rightarrow \mathbb{R}_{++}^{n}$ be a WPO, COV, and IIA bargaining solution that is not an iterated Nash bargaining solution. Then we cannot prove in ZFC that the sets $L_{\varphi}, D_{\varphi}$ and $R_{\varphi}$ are definable sets.

Proof Let $\varphi: \mathcal{F} \rightarrow \mathbb{R}_{++}^{n}$ be a WPO, COV, and IIA bargaining solution, and assume that it is not an iterated Nash bargaining solution. Then, by Theorem 3.9 there exists a (unique) iterated Nash bargaining solution $N$ such that $\varphi$ is a strict refinement of $N$ and $R_{\varphi} \cap D_{N}$ is dense in $D_{N}$.

We show that $R_{\varphi}$ is not definable. Define the operation $\rho: D_{N} \rightarrow \mathbb{R}^{n}$ by

$$
\rho\left(x_{1}, \ldots, x_{n}\right):=\left(\ln \left(x_{1}\right), \ldots, \ln \left(x_{n}\right)\right) .
$$

Write $H:=\rho\left(D_{N}\right)$. It is straightforward to check that $H$ is a closed and additive, and hence linear, subspace of $\mathbb{R}^{n}$. Furthermore, $L:=\rho\left(L_{\varphi} \cap D_{N}\right), D:=\rho\left(D_{\varphi}\right)$ and $R:=\rho\left(R_{\varphi} \cap D_{N}\right)$ form an LDR decomposition of $H$, and $R$ is dense in $H$. So, by Lemma 4.3 there is a line $P$ in $H$ through the origin such that $P \cap R$ is dense in $P$. Then by Lemma 4.2 we know that $P$ is not measurable. Hence, by the above line of reasoning, we cannot show in ZFC that $P$ is definable.

On the other hand, suppose that $R_{\varphi}$ is definable. Then, because $D_{N}$ is definable, also $R_{\varphi} \cap D_{N}$ is definable. Hence, also $P=\rho\left(R_{\varphi} \cap D_{N}\right)$ is definable. Contradiction. Hence, $R_{\varphi}$ cannot be definable.

Since the graph of a bargaining solution $\varphi$ is not an object in Euclidean space we cannot, as is customary in most cases, directly prove that the graph of $\varphi$ is not definable, simply because the notion of definability does not apply to such an object as the graph of a bargaining solution. Nevertheless, Theorem 4.9 implies the following. Suppose we wish to construct WPO, COV and IIA bargaining solutions on domain $\mathcal{F}$ by means of their encoding in quadruples $\left(\alpha, L_{\varphi}, D_{\varphi}, R_{\varphi}\right)$. Further suppose we only wish to describe the ingredients of a quadruple by means of set-theoretic formulae of the form $\Phi\left(t, r, \alpha_{1}, \alpha_{2}, \ldots\right)$. Then the only solutions we can construct are the iterated Nash bargaining solutions. All other solution necessarily involve the use of sets that cannot be expressed in terms of such formulae. This shows in a very strong sense that we cannot "explicitly" define WPO, COV and IIA bargaining solutions of type II. 


\section{Consequences of the characterizations}

In this section we discuss some consequences of the characterizations in Sect. 3.

\subsection{Nash bargaining solutions}

We show that the class of Nash bargaining solutions is characterized by WPO, COV, IIA and $\mathrm{CON}$ on any domain $\mathcal{D}$ containing the domain of finite bargaining problems and contained in the domain of all compact bargaining problems. ${ }^{19}$

Thus, in this section we assume that $\mathcal{D}$ is a domain with $\mathcal{F} \subset \mathcal{D} \subset \mathcal{C}$. Let $\varphi: \mathcal{D} \rightarrow$ $\mathbb{R}_{++}^{n}$ be a WPO, COV and IIA bargaining solution. With a slight abuse of notation we will not distinguish between $\varphi$ itself and its restriction $\varphi_{\mathcal{F}}$ to the domain $\mathcal{F}$ of all finite bargaining problems because, by IIA, there is a simple one-to-one correspondence between $\varphi$ and $\varphi_{\mathcal{F}}$. We need the following observation.

Lemma 5.1 Let $\varphi: \mathcal{D} \rightarrow \mathbb{R}_{++}^{n}$ be a WPO, COV and IIA bargaining solution. Then $\varphi$ is a Nash bargaining solution if and only if $D_{\varphi}=I_{\varphi}$.

\section{Proof}

A. Suppose that $D_{\varphi}=I_{\varphi}$. Let $\left(\alpha, L_{\varphi}, D_{\varphi}, R_{\varphi}\right)$ be the characteristic quadruple of $\varphi$. From Lemma A.5,

$$
\left(\alpha, L_{\varphi}, D_{\varphi}, R_{\varphi}\right)=\left(\alpha, \phi, I_{\varphi}, \phi\right)=\left(\alpha, \phi, I_{\alpha}, \phi\right)
$$

It is straightforward to check that $N^{\alpha}$ has the same characteristic quadruple. Hence, by Theorem 3.6, $\varphi=N^{\alpha}$.

B. Suppose that $\varphi$ is a Nash bargaining solution. Then $I_{\varphi} \supset D_{\varphi}$. Take $x \in I_{\varphi}$. We show that $x \in D_{\varphi}$. By assumption there is a non-zero vector $\alpha \geq 0$ such that $\varphi=N^{\alpha}$. Then clearly $I_{\varphi}=I_{N^{\alpha}}$. Hence, $\varphi(x, e)=N^{\alpha}(x, e)=\{x, e\}$.

Now we can prove the following characterization of Nash bargaining solutions.

Theorem 5.2 Let $\varphi: \mathcal{D} \rightarrow \mathbb{R}_{++}^{n}$ be a bargaining solution. Then $\varphi$ satisfies WPO, $\mathrm{COV}$, IIA and $\mathrm{CON}$ if and only if $\varphi$ is a Nash bargaining solution.

Proof We only prove the only-if direction. Let $\varphi$ satisfy the axioms in the theorem. By Lemma 5.1 it suffices to show that $D_{\varphi}=I_{\varphi}$. Take an $x \in I_{\varphi}$. Then, for any positive $\lambda<1, \varphi(\lambda x, e)=\{e\}$ by Lemma 3.2. Hence, by CON, $e \in \varphi(x, e)$. Conversely, for any $\lambda>1, \varphi(\lambda x, e)=\{\lambda x\}$ by Lemma 3.2. Hence, by CON, $x \in \varphi(x, e)$.

In different but equivalent formulations this result was also derived in Kaneko and Nakamura (1979) and Naumova and Yanovskaya (2001).

Imposing SYM instead of CON singles out the symmetric Nash bargaining solution $N^{\alpha}$ with $\alpha_{i}=1 / n$ for every $i$.

\footnotetext{
19 With some slight modifications this assumption can easily be relaxed, e.g., by assuming that $\mathcal{D}$ contains all finitely generated comprehensive problems.
} 
Theorem 5.3 Let $\varphi: \mathcal{D} \rightarrow \mathbb{R}_{++}^{n}$ be a bargaining solution. Then $\varphi$ satisfies WPO, COV, IIA and SYM if and only if $\varphi$ is the symmetric Nash bargaining solution.

Proof We only prove the if-direction. Let $\varphi$ be a bargaining solution with these properties. Obviously, $\alpha_{i}=1 / n$ for all $i$. Take $x \in I_{\varphi}$. It is sufficient to prove that $x \in D_{\varphi}$. It is not difficult (cf. Xu and Yoshihara 2006) to find an $a \in \mathbb{R}_{++}^{n}$ such that $a=a e$ can be obtained from $a x$ by a permutation of coordinates. Consider the finite bargaining problem $B$ consisting of $a x$ and all vectors obtainable from $a x$ by a permutation of coordinates. By SYM, $\varphi(B)=B$. In particular, $a, a x \in \varphi(B)$. By COV and IIA, $\varphi(x, e)=\{x, e\}$, hence $x \in D_{\varphi}$.

This result was also derived in Mariotti (1998a) and Xu and Yoshihara (2006).

\subsection{Compact domain}

In this subsection we show that any WPO, COV and IIA bargaining solution on the domain $\mathcal{C}$ of all compact bargaining problems is an iterated Nash bargaining solution. As already established, under the Axiom of Determinacy a WPO, COV and IIA bargaining solution defined on any domain $\mathcal{D}$ that includes $\mathcal{F}$ is an iterated Nash bargaining solution. Theorem 3.1 of Naumova and Yanovskaya (2001) shows that on the domain $\mathcal{C}$ this remains true even without the Axiom of Determinacy. Their proof is based on a theorem of Birkhoff (see the next section for a discussion of that theorem). Using Theorem 3.9 we show the above result by arguing directly that WPO, COV and IIA bargaining solutions of type II cannot be extended to the domain $\mathcal{C}$ of all compact bargaining problems.

Theorem 5.4 A bargaining solution $\varphi$ on the domain $\mathcal{C}$ of all compact bargaining problems satisfies WPO, COV and IIA if and only if it is an iterated Nash bargaining solution.

Proof It is clear that any iterated Nash bargaining solution satisfies WPO, COV and IIA. Let $\varphi$ be a WPO, COV and IIA bargaining solution on $\mathcal{C}$. Let $N$ be the iterated Nash bargaining solution defined in the proof of Theorem 3.9. It suffices to show that $R_{\varphi} \cap D_{N}$ cannot be dense in $D_{N}$.

Suppose $R_{\varphi} \cap D_{N}$ is dense in $D_{N}$. We derive a contradiction. Write $L:=L_{\varphi} \cap D_{N}$ and $R:=R_{\varphi} \cap D_{N}$. Clearly $D_{N}$ is an Abelian multiplicative group, and $L$ and $R$ are dense subsets of $D_{N}$. So, at least there exists an $r \in R$. Since $L$ is dense in $D_{N}$ we can take an $l_{1} \in L$ with $d\left(\rho(r), \rho\left(l_{1}\right)\right)<\frac{1}{2}$, where $d(x, y)$ denotes the Euclidean distance between $x$ and $y$, and $\rho: I_{\alpha} \rightarrow H_{\alpha}$ is the isomorphism defined in Sect. 4.2.

Recursively, suppose for $m \in \mathbb{N}, m \geq 2$ that $l_{1}, \ldots, l_{m-1}$ have been chosen in $L$. Since $r \prod_{k=1}^{m-1} l_{k}^{-1}$ is an element of $D_{N}$, we can take an $l_{m} \in L$ such that

$$
d\left(\rho\left(r \prod_{k=1}^{m-1} l_{k}^{-1}\right), \rho\left(l_{m}\right)\right)<\left(\frac{1}{2}\right)^{m} .
$$


Write $L_{m}:=\prod_{k=1}^{m} l_{m}$ for each $m \in \mathbb{N}$. Then, by translation invariance of the Euclidean distance and preservation of group structure by $\rho$,

$$
\begin{aligned}
d\left(\rho(r), \rho\left(L_{m}\right)\right) & =d\left(\rho(r), \sum_{k=1}^{m} \rho\left(l_{k}\right)\right)=d\left(\rho(r)-\sum_{k=1}^{m-1} \rho\left(l_{k}\right), \rho\left(l_{m}\right)\right) \\
& =d\left(\rho\left(r \prod_{k=1}^{m-1} l_{k}^{-1}\right), \rho\left(l_{m}\right)\right)<\left(\frac{1}{2}\right)^{m} .
\end{aligned}
$$

In particular

$$
B:=\left\{e, r, L_{1}, L_{2}, \ldots\right\}
$$

is a compact set. Thus, $B \in \mathcal{C}$. We show that $\varphi(B)$ must be empty, which contradicts the assumption that $\varphi$ is a bargaining solution. Firstly notice that $\varphi\left(e, L_{1}\right)=\left\{L_{1}\right\}$ because $L_{1}=l_{1}$ is an element of $L \subset L_{\varphi}$. Hence, $e \notin \varphi(B)$ by IIA. Secondly notice that $\varphi(e, r)=\{e\}$ because $r$ is an element of $R \subset R_{\varphi}$. Hence, $r \notin \varphi(B)$ by IIA. Thirdly, for any $m \in \mathbb{N}$, by COV we have

$$
\varphi\left(L_{m}, L_{m+1}\right)=L_{m} \cdot \varphi\left(e, l_{m+1}\right)=L_{m} \cdot\left\{l_{m+1}\right\}=\left\{L_{m+1}\right\}
$$

because $l_{m+1}$ is an element of $L \subset L_{\varphi}$. Hence, for any $m \in \mathbb{N}, L_{m} \notin \varphi(B)$ by IIA, and none of the elements of $B$ can be an element of $\varphi(B)$.

Theorem 5.4 extends the main results in Zhou (1996) and Denicolò and Mariotti (2000), who show that any single-valued bargaining solution satisfying WPO, COV and IIA must select from some Nash bargaining solution. From Theorem 5.4 it follows that such a solution must be a single-valued iterated Nash bargaining solution. It follows from Theorem 5.2 that these solutions cannot be continuous.

\section{The additive case and Birkhoff's theorem}

The results in this section are the additive counterparts of the constructions in the previous sections. One could derive the third characterization in Sect. 3 and Theorem 4.4 by first proving the results in this section and then lifting these results to the bargaining context. However, since this would require a long pre-discussion and several extra lifting results before we could present our main observations, we decided not to pursue this avenue, and simply to present the results on additive groups separately without any further proofs.

We first formulate the Birkhoff Theorem (Birkhoff 1948). An ordering on $\mathbb{R}^{n}$ is a binary relation $\preceq$ on $\mathbb{R}^{n}$ that is complete, reflexive, and transitive. We write $x \prec y$ if $x \preceq y$ and not $y \preceq x$. We write $x \sim y$ if $x \preceq y$ and $y \preceq x$. An ordering $\preceq$ is translation invariant if for all $x, y, z \in \mathbb{R}^{n}, x \preceq y$ holds whenever $x+z \preceq y+z$. The ordering is positive homogeneous if for all $x, y \in \mathbb{R}^{n}$ and all $\lambda>0, x \preceq y$ holds whenever $\lambda x \preceq \lambda y$. 
Let $\beta=\left(\beta^{1}, \ldots, \beta^{m}\right)$ a sequence of orthogonal vectors $\beta \in \mathbb{R}^{n}$. For $x, y \in \mathbb{R}^{n}$, the inner product $\langle x, y\rangle$ is defined by $\langle x, y\rangle:=\sum_{i} x_{i} y_{i}$. The binary relation $\preceq_{\beta}$ on $\mathbb{R}^{n}$ is defined as follows. For every $x, y \in \mathbb{R}^{n}, x \preceq y$ when either $\left\langle\beta^{k}, x\right\rangle=\left\langle\beta^{k}, y\right\rangle$ for all $1 \leq k \leq m$, or there is an $l, 1 \leq l \leq m$ with $\left\langle\beta^{k}, x\right\rangle=\left\langle\beta^{k}, y\right\rangle$ for all $k<l$ and $\left\langle\beta^{l}, x\right\rangle<\left\langle\beta^{l}, y\right\rangle$.

Theorem 6.1 (Birkhoff) An ordering $\preceq$ is translation invariant and positive homogeneous if and only if there is a sequence $\beta=\left(\beta^{1}, \ldots, \beta^{m}\right)$ of orthogonal vectors such that $\preceq=\preceq \beta$.

Using the techniques we developed in this paper we can prove the following generalization of Birkhoff's theorem. For a sequence $\beta=\left(\beta^{1}, \ldots, \beta^{m}\right)$ of orthogonal vectors, write

$$
H_{\beta}:=\left\{x \in \mathbb{R}^{n} \mid\left\langle\beta^{k}, x\right\rangle=0 \text { for all } 1 \leq k \leq m\right\} .
$$

Clearly $H_{\beta}$ is a linear subspace of $\mathbb{R}^{n}$. Let $L, D$ and $R$ form an additive LDR decomposition of $H_{\beta}$. We say that the ordering $\preceq$ is induced by the quadruple $(\beta, L, D, R)$ if $x \preceq y$ holds precisely when one of the following two cases is valid.

(1) $x \prec \beta \quad y$

(2) $x \sim_{\beta} y$ (so $y-x \in H_{\beta}$ ), and $y-x \in R$.

Theorem 6.2 An ordering $\preceq$ is translation invariant if and only if there exists a sequence $\beta$ of orthogonal vectors and an LDR decomposition $L, D$ and $R$ of $H_{\beta}$ such that $\preceq$ is induced by the quadruple $(\beta, L, D, R)$ and either $R=\phi$ or $R$ is dense in $H_{\beta}$.

The correspondence between translation invariant orderings $\preceq$ and quadruples ( $\beta, L, D, R$ ) with either $R=\phi$ or $R$ dense in $H_{\beta}$ is one-to-one and onto up to scalar multiplication of the orthogonal vectors in the sequence $\beta$.

Proof of Birkhoff's theorem. It is straightforward to show that, for a sequence $\beta=$ $\left(\beta^{1}, \ldots, \beta^{m}\right)$ of orthogonal vectors, $\leq_{\beta}$ is translation invariant and positive homogeneous. We show the other implication.

Suppose that $\preceq$ is translation invariant and positive homogeneous. Let $(\beta, L, D, R)$ be the associated quadruple. We show that $R$ is empty. Suppose it is not. Then $R$ is dense in $H_{\beta}$. So, by Lemma 4.3 there is a line $P$ in $H_{\beta}$ through the origin such that $P \cap R$ is dense in $P$. Take a point $r \in P \cap R$. Notice that $r \neq 0$. Since $L=-R, P \cap L$ is also dense in $P$. So, there must be a $\mu>0$ such that $\mu r \in L$. Then however $0 \prec r$ and $\mu r \prec 0$. This violates positive homogeneity.

Theorem 6.3 Assume the Axiom of Determinacy. Then an ordering $\preceq$ is translation invariant if and only if there is a sequence $\beta=\left(\beta^{1}, \ldots, \beta^{m}\right)$ of orthogonal vectors such that $\preceq=\preceq \beta$.

Proof Suppose that $\preceq$ is translation invariant. Let $(\beta, L, D, R)$ be the associated quadruple. We show that $R$ is empty. Suppose it is not. Then $R$ is dense in $H_{\beta}$. So, by Lemma 4.3 there is a line $P$ in $H_{\beta}$ through the origin such that $P \cap R$ is dense in $P$. This contradicts Theorems 4.2 and 4.1. 
If $x \prec y$ holds for all $x, y \in \mathbb{R}^{n}$ with $x<y$ we say that $\preceq$ satisfies Weak Pareto Optimality (WPO). For WPO orderings we obtain the same set of results, with the only proviso that the first vector $\beta^{1}$ in the sequence is non-zero and nonnegative.

INDEPENDENCE OF AXIOMS. In fact these results show that the independence of the axiom of positive homogeneity in the characterization of orderings of the form $\preceq_{\beta}$ is debatable. When we accept the Axiom of Choice, positive homogeneity is indeed an independent axiom. However, when we accept the Axiom of Determinacy, Theorem 6.3 shows that the axiom of positive homogeneity can be dropped from the formulation of Birkhoff's theorem.

ADDITIVE FUNCTIONS. As a final curiosity, we observe that under the Axiom of Determinacy, every additive function on the reals is necessarily linear. However, under the Axiom of Choice, there are many more non-linear additive functions than linear functions.

\section{Discussion and conclusion}

In this final section we discuss independence of the basic axioms, and we further discuss related literature. We end with some concluding remarks.

\subsection{Independence of the axioms}

We show the independence of the basic axioms used in this paper.

Not WPO The bargaining solution $\varphi: \mathcal{F} \rightarrow \mathbb{R}_{++}^{n}$ defined by $\varphi(F):=F$ for all $F \in \mathcal{F}$ satisfies IIA, COV and CON, but not WPO.

Not IIA The bargaining solution WPO: $\mathcal{F} \rightarrow \mathbb{R}_{++}^{n}$ defined by, for all $F \in \mathcal{F}$,

$$
\mathrm{WPO}(F):=\{x \in F \mid x<y \text { implies that } y \notin F\}
$$

satisfies WPO, COV and CON, but not IIA.

Not COV The bargaining solution $\varphi: \mathcal{F} \rightarrow \mathbb{R}_{++}^{n}$ defined by, for all $F \in \mathcal{F}$,

$$
\varphi(F):=\left\{x \in F \mid \sum_{i} x_{i} \geq \sum_{i} y_{i} \text { for all } y \in F\right\}
$$

satisfies WPO, IIA and CON, but not COV.

Not CON Bargaining solutions of Type II as constructed in Sect. 4.2, or iterated Nash bargaining solutions that are not Nash bargaining solutions, satisfy WPO, COV and IIA but not CON.

Note that the first three counterexamples can be extended to any domain $\mathcal{D}$ with $\mathcal{F} \subset \mathcal{D} \subset \mathcal{C}$. As for the continuity axiom, on the domain $\mathcal{C}$ the independence follows from Theorems 5.2 and 5.4. 


\subsection{Related literature}

We continue and extend the discussion started in Sect. 2.

Kaneko (1980) characterizes the symmetric Nash (multi-valued) bargaining solution on the domain of all compact $n$-person bargaining problems by the axioms PO, IIA, COV, SYM, and CON. A recent improvement of this result is obtained by Xu and Yoshihara (2006), who are able to drop CON from this set of axioms. Both results follow from Theorem 5.3, which, in turn, is a relatively straightforward consequence of the results in Sect. 3.

Mariotti (1998a) characterizes the two-person ${ }^{20}$ symmetric Nash bargaining solution on the domain of finite bargaining problems by WPO, COV, SYM, and an axiom called 'Pareto monotonicity', which is implied by WPO and IIA. Hence, this characterization follows from Theorem 5.3. Theorem 4.3 in Mariotti (1998a) about the symmetric two-person Nash bargaining solution on a larger domain also follows from our Theorem 5.3. Likewise, Observation 5.3.1 in Mariotti (1998a) about the nonexistence of a two-person symmetric single-valued solution with the usual properties on a domain of compact connected problems follows from this result.

Zhou (1996) shows that, on the domain of compact $n$-person bargaining problems, any single-valued solution satisfying COV, IIA, and strict individual rationality refines some Nash bargaining solution. As in Roth (1977) it can be shown that these conditions imply WPO, and thus Zhou's result follows from our basic results in Sect. 3. Moreover, Theorem 5.4 shows exactly how this refinement works. This also applies to Denicolò and Mariotti (2000), who derive a similar result as Zhou (1996) under somewhat stronger conditions. Their proof is based on a result of d'Aspremont (1985), characterizing orderings.

For a discussion of Naumova and Yanovskaya (2001) see, in particular, Sect. 5.2. For the relation with Kaneko and Nakamura (1979) see Sect. 5.1.

To our knowledge the papers mentioned so far are the ones that are most directly related to our work. Herrero (1989) and Maschler et al. (1988) consider the symmetric Nash bargaining solution on domains of compact bargaining problems from a more geometrical point of view, and complement it by a noncooperative game (Herrero 1989) or by a dynamic system (Maschler et al. 1988). Conley and Wilkie (1996) and Mariotti (1998b) study a different single-valued extension of the symmetric Nash bargaining solution to non-convex bargaining problems.

\subsection{Concluding remarks}

The basic distinguishing features of the present paper are the possible non-convexity of the bargaining problems under consideration and the possible non-continuity of bargaining solutions. Omitting continuity as a basic requirement for solutions has enabled us to give a detailed analysis of the consequences of the basic conditions in this paper, viz. WPO, COV and IIA. The generality of the analysis is indicated

20 The paper also indicates how to extend this result to the $n$-person case. 
by the fact that much of what is known about bargaining solutions under these three conditions follows from it.

Our discussion concerning the use of the Axiom of Determinacy versus the Axiom of Choice may be interpreted as making a case for the former. Accepting the Axiom of Determinacy leaves the class of iterated Nash bargaining solutions on any domain containing the domain of finite—or finitely generated—bargaining problems, allowing considerably more flexibility than is obtained by imposing continuity. The Axiom of Choice allows for many more bargaining solutions but these can only be obtained by a construction which cannot be carried out in practice. This point of view is corroborated by the fact that these additional solutions are not definable.

Acknowledgements We thank participants of several seminars for useful comments. In particular, we thank Luc Lauwers, Marco Mariotti, Natalia Naumova, Peter Wakker, Jan Mycielski, and William Zame for comments on earlier drafts of this paper. We are indebted to Wim Veldman and Arnoud van Rooij of the Mathematical Institute, Radboud University Nijmegen, The Netherlands, for pointing our attention to the Axiom of Determinacy. Also, the comments of two anonymous referees helped to improve the paper considerably.

Open Access This article is distributed under the terms of the Creative Commons Attribution Noncommercial License which permits any noncommercial use, distribution, and reproduction in any medium, provided the original author(s) and source are credited.

\section{A Appendix: basic facts and proofs for the first characterization}

Proof of Lemma 3.1 Because all coordinates of $x$ are strictly positive, by WPO and the non-emptiness of $\varphi(x, \lambda y)$ there exists a $\mu>0$ such that $\varphi(x, \lambda y)=\{x\}$ for all $0<\lambda<\mu$. Hence, $\lambda(x, y)>0$. Similarly, because all coordinates of $y$ are positive, there is a $\lambda>0$ such that $\varphi(x, \lambda y)=\{\lambda y\}$. Hence $\lambda(x, y)<\infty$.

Proof of Lemma 3.2 We only show the first statement. Let $\lambda<\lambda(x, y)$ and suppose that $\lambda y \in \varphi(x, \lambda y)$. Take a $\mu$ with $\lambda<\mu<\lambda(x, y)$. Notice that $\varphi(\lambda y, \mu y)=\{\mu y\}$ by WPO and the non-emptiness of $\varphi(\lambda y, \mu y)$. Thus, $\mu y \in \varphi(x, \mu y)$ by transitivity of $\varphi$, which contradicts the definition of $\lambda(x, y)$. Hence, $\lambda y \notin \varphi(x, \lambda y)$ and then $\varphi(x, \lambda y)=\{x\}$ by non-emptiness.

Throughout the remainder of this appendix, let $\varphi: \mathcal{F} \rightarrow \mathbb{R}_{++}^{n}$ be a bargaining solution that satisfies WPO, IIA and COV.

Clearly, by WPO, $x$ itself is an element of the generalized indifference curve $I_{\varphi}(x)$, and each ray emanating from the origin into $\mathbb{R}_{++}^{n}$ intersects this curve at most once. We show that each ray also intersects this curve at least once.

We need a few more facts about the generalized indifference curve. First, generalized indifference curves partition $\mathbb{R}_{++}^{n}$. Another way of saying this is that generalized indifference curves are the equivalence classes of an equivalence relation.

Lemma A.1 Let $x, y \in \mathbb{R}_{++}^{n}$ such that $y \in I_{\varphi}(x)$. Then $I_{\varphi}(x)=I_{\varphi}(y)$.

Proof

(a) Suppose that $z \in I_{\varphi}(y)$. We show that $z \in I_{\varphi}(x)$. Take $\lambda>0$. 
(a1) Suppose $\lambda>1$. Then $\varphi(y, \sqrt{\lambda} z)=\{\sqrt{\lambda} z\}$ by Lemma 3.2. So, by COV, $\varphi(\sqrt{\lambda} y, \lambda z)=\{\lambda z\}$. However, since $y \in I_{\varphi}(x), \varphi(x, \sqrt{\lambda} y)=\{\sqrt{\lambda} y\}$. Hence, $\varphi(x, \lambda z)=\{\lambda z\}$ by transitivity.

(a2) Suppose $\lambda<1$. Then $\varphi(y, \sqrt{\lambda} z)=\{y\}$ by Lemma 3.2. So, by COV, $\varphi(\sqrt{\lambda} y, \lambda z)=\{\sqrt{\lambda} y\}$. However, since $y \in I_{\varphi}(x), \varphi(x, \sqrt{\lambda} y)=\{x\}$. Hence, $\varphi(x, \lambda z)=\{x\}$ by transitivity.

(b) By (a1) and (a2), $\lambda(x, z)=1$ and hence $I_{\varphi}(y) \subset I_{\varphi}(x)$. We show that $x \in I_{\varphi}(y)$. Take $\lambda>0$.

(b1) Suppose $\lambda>1$. Then, since $y \in I_{\varphi}(x), \varphi\left(\frac{1}{\lambda} y, x\right)=\{x\}$. Hence, $\varphi(y, \lambda x)=$ $\{\lambda x\}$ by COV.

(b2) Suppose $\lambda<1$. Then, since $y \in I_{\varphi}(x), \varphi\left(\frac{1}{\lambda} y, x\right)=\left\{\frac{1}{\lambda} y\right\}$. Hence, $\varphi(y, \lambda x)=$ $\{y\}$ by COV.

By (b1) and (b2), $\lambda(y, x)=1$ and $x \in I_{\varphi}(y)$.

Lemma A.2 For every $x \in \mathbb{R}_{++}^{n}, I_{\varphi}(x)$ is closed.

Proof Let $\left(y_{m}\right)_{m \in \mathbb{N}}$ be a sequence in $I_{\varphi}(x)$ that converges to $y$.

(a) Take $\lambda>1$. Since $y_{m} \rightarrow y$ there must be a $k \in \mathbb{N}$ such that $y_{k}<\lambda y$. Then there exists $\mu>1$ such that $\mu y_{k}<\lambda y$ also holds. Now, since $\varphi\left(x, \mu y_{k}\right)=\left\{\mu y_{k}\right\}$ and $\varphi\left(\mu y_{k}, \lambda y\right)=\{\lambda y\}$, we have $\varphi(x, \lambda y)=\{\lambda y\}$ by transitivity.

(b) Take $\lambda<1$. Since $y_{m} \rightarrow y$ there must be a $k \in \mathbb{N}$ such that $y_{k}>\lambda y$. Then there exists $\mu<1$ such that $\mu y_{k}>\lambda y$ also holds. Now, since $\varphi\left(x, \mu y_{k}\right)=\{x\}$ and $\varphi\left(\mu y_{k}, \lambda y\right)=\left\{\mu y_{k}\right\}$, we have $\varphi(x, \lambda y)=\{x\}$ by transitivity.

From (a) and (b) it follows that $\lambda(x, y)=1$ and hence $y \in I_{\varphi}(x)$.

Lemma A.3 Let $a \in \mathbb{R}_{++}^{n}$ and $y \in I_{\varphi}(x)$. Then ay $\in I_{\varphi}(a x)$.

Proof Follows immediately from COV.

Lemma A.4 Let $a \in \mathbb{R}_{++}^{n}$. Suppose that ax $\in I_{\varphi}(x)$. Then $a^{q} x \in I_{\varphi}(x)$ for all $q \in \mathbb{Q}$.

Proof Take $a \in \mathbb{R}_{++}^{n}$ and $q \in \mathbb{Q}$. Suppose that $a x \in I_{\varphi}(x)$.

(a) First we show that $a^{k} x \in I_{\varphi}(x)$ for all $k \in \mathbb{N}$. Since $x$, ax $\in I_{\varphi}(x)$, this is true for $k=0,1$. Suppose $a^{k} x \in I_{\varphi}(x)$ for $0 \leq k \leq l$. Since $I_{\varphi}(a x)=I_{\varphi}(x)$ by Lemma A.1, we have

$$
a^{l+1} x=a \cdot a^{l} x \in I_{\varphi}(a x)=I_{\varphi}(x)
$$

by Lemma A.3. Hence, $a^{k} x \in I(x)$ for all $k \in \mathbb{N}$.

(b) Next, take a $k \in \mathbb{N}, k \neq 0$. We show that $a^{\frac{1}{k}} x \in I_{\varphi}(x)$.

(b1) Take $\lambda>1$. Suppose that $x \in \varphi\left(x, \lambda a^{\frac{1}{k}} x\right)$. Then, because of COV, multiplication by $\lambda^{l} a^{\frac{l}{k}}$ shows that

$$
\lambda^{l} a^{\frac{l}{k}} x \in \varphi\left(\lambda^{l} a^{\frac{l}{k}} x, \lambda^{l+1} a^{\frac{l+1}{k}} x\right)
$$


for all $0 \leq l \leq k-1$. So, iterated application of transitivity yields that $x \in \varphi\left(x, \lambda^{k} a x\right)$. This contradicts the assumption that $a x \in I_{\varphi}(x)$. Hence, $\varphi\left(x, \lambda a^{\frac{1}{k}} x\right)=\left\{\lambda a^{\frac{1}{k}} x\right\}$.

(b2) Take $\lambda<1$. Suppose that $\lambda a^{\frac{1}{k}} x \in \varphi\left(x, \lambda a^{\frac{1}{k}} x\right)$. Then, because of COV, multiplication by $\lambda^{l} a^{\frac{l}{k}}$ shows that

$$
\lambda^{l+1} a^{\frac{l+1}{k}} x \in \varphi\left(\lambda^{l} a^{\frac{l}{k}} x, \lambda^{l+1} a^{\frac{l+1}{k}} x\right)
$$

for all $0 \leq l \leq k-1$. So, $\lambda^{k} a x \in \varphi\left(x, \lambda^{k} a x\right)$ by transitivity. This contradicts the assumption that $a x \in I_{\varphi}(x)$. Hence, $\varphi\left(x, \lambda a^{\frac{1}{k}} x\right)=\{x\}$.

From (b1) and (b2) it follows that $a^{\frac{1}{k}} x \in I_{\varphi}(x)$.

(c) Since $a x \in I_{\varphi}(x)$, we know that $x \in I_{\varphi}(a x)$ by Lemma A.1. Hence, by Lemma A.3, also $a^{-1} x \in I_{\varphi}\left(a^{-1} a x\right)=I_{\varphi}(x)$.

From (a), (b) and (c) it follows that $a^{q} x \in I_{\varphi}(x)$.

Lemma A.5 Let $\alpha=\left(\alpha_{1}, \ldots, \alpha_{n}\right)$ be as defined in Sect. 3.1. Then, for all $x, y \in \mathbb{R}_{++}^{n}$ we have $y \in I_{\varphi}(x)$ if and only if

$$
\prod_{i} x_{i}^{\alpha_{i}}=\prod_{i} y_{i}^{\alpha_{i}}
$$

Proof Define $H$ as the set of vectors $y \in \mathbb{R}_{++}^{n}$ with $\prod_{i} y_{i}^{\alpha_{i}}=1$. By COV it suffices to show that $I_{\varphi}(e)=H$. We prove this as follows. Write $b^{j}:=\lambda\left(a^{j}\right) a^{j}$. Let $B$ be the collection of vectors in $\mathbb{R}_{++}^{n}$ that can be written as

$$
\prod_{j=1}^{n}\left(b^{j}\right)^{\mu_{j}}
$$

with $\mu_{1}, \ldots, \mu_{n} \in \mathbb{R}$. First we show that $B=H$. Then we show that $B \subset I_{\varphi}(e)$. Finally we argue that $I_{\varphi}(e)=H$.

A1. Take $b=\prod_{j=1}^{n}\left(b^{j}\right)^{\mu_{j}} \in B$. Then

$$
\begin{aligned}
\prod_{i} b_{i}^{\alpha_{i}} & =\prod_{i}\left(\prod_{j}\left(b_{i}^{j}\right)^{\mu_{j}}\right)^{\alpha_{i}} \\
& =\prod_{i}\left(\prod_{j}\left(\lambda\left(a^{j}\right) \cdot a_{i}^{j}\right)^{\mu_{j}}\right)^{\alpha_{i}} \\
& =\prod_{i} \prod_{j}\left(\lambda\left(a^{j}\right) \cdot a_{i}^{j}\right)^{\alpha_{i} \mu_{j}} \\
& =\prod_{j} \prod_{i} \lambda\left(a^{j}\right)^{\alpha_{i} \mu_{j}} \cdot \prod_{i} \prod_{j}\left(a_{i}^{j}\right)^{\alpha_{i} \mu_{j}}
\end{aligned}
$$




$$
\begin{aligned}
& =\prod_{j} \lambda\left(a^{j}\right)^{\mu_{j} \sum_{i} \alpha_{i}} \cdot \prod_{i}\left(a_{i}^{i}\right)^{\alpha_{i} \mu_{i}} \\
& =\prod_{j} \lambda\left(a^{j}\right)^{\mu_{j}} \cdot \prod_{i}\left(\frac{1}{2}\right)^{\alpha_{i} \mu_{i}} \\
& =\prod_{j} \lambda\left(a^{j}\right)^{\mu_{j}} \cdot \prod_{i}\left(\frac{1}{2}\right)^{\mu_{i} \cdot{ }^{2} \log \lambda\left(a^{i}\right)} \\
& =\prod_{j} \lambda\left(a^{j}\right)^{\mu_{j}} \cdot\left(\prod_{i} \lambda\left(a^{i}\right)^{\mu_{i}}\right)^{-1} \\
& =1,
\end{aligned}
$$

where the fifth equality follows from the definition of $a^{j}$, while the sixth equality follows from both the definition of $a^{j}$ and Lemma 3.3. Hence, $b \in H$.

A2. Conversely, let $y \in H$. So, $y \in \mathbb{R}_{++}^{n}$ and $\prod_{i} y_{i}^{\alpha_{i}}=1$. We show that there are $\mu_{1}, \ldots, \mu_{n} \in \mathbb{R}$ such that for all $i$

$$
y_{i}=\prod_{j=1}^{n}\left(b_{i}^{j}\right)^{\mu_{j}} .
$$

Write $z_{i}:={ }^{2} \log y_{i}$. Since $b_{i}^{j}=\lambda\left(a^{j}\right) a_{i}^{j}$ by definition, we have $b_{i}^{j}=\lambda\left(a^{j}\right)$ for all $j \neq i$ and $b_{i}^{i}=\frac{1}{2} \lambda\left(a^{i}\right)$. Thus, taking base 2 logarithms in (1) shows that we have to find $\mu_{1}, \ldots, \mu_{n} \in \mathbb{R}$ such that for all $i$

$$
z_{i}=-\mu_{i}+\sum_{j} \alpha_{j} \mu_{j}
$$

Write

$$
A=\left[\begin{array}{llll}
\alpha_{1}-1 & \alpha_{2} & \cdots & \alpha_{n} \\
\alpha_{1} & \alpha_{2}-1 & \cdots & \alpha_{n} \\
\vdots & \vdots & \ddots & \vdots \\
\alpha_{1} & \alpha_{2} & \cdots & \alpha_{n}-1
\end{array}\right], \quad \mu=\left[\begin{array}{l}
\mu_{1} \\
\vdots \\
\mu_{n}
\end{array}\right] \quad \text { and } z=\left[\begin{array}{l}
z_{1} \\
\vdots \\
z_{n}
\end{array}\right]
$$

We show that $z=A \mu$ has a solution. Let $Z$ be the set of vectors $x \in \mathbb{R}^{n}$ with $\sum_{i} \alpha_{i} x_{i}=0$. Since $\sum_{i} \alpha_{i}=1$, we have $\operatorname{dim}(Z)=n-1$. Further, from A1, each vector of the form $\prod_{j=1}^{n}\left(b^{j}\right)^{\mu_{j}}$ is an element of $H$. Taking base 2 logarithms this implies that each vector of the form $A \mu$ is an element of $Z$. Thus, $\operatorname{Im}(A)$ is a subspace of $Z$. However, subtracting the first row of $A$ from its other rows yields 
the matrix

$$
B=\left[\begin{array}{lllll}
\alpha_{1}-1 & \alpha_{2} & \alpha_{3} & \cdots & \alpha_{n} \\
-1 & -1 & 0 & \cdots & 0 \\
-1 & 0 & -1 & \cdots & 0 \\
\vdots & \vdots & \vdots & \ddots & \vdots \\
-1 & 0 & 0 & \cdots & -1
\end{array}\right]
$$

So, $\operatorname{dim}(\operatorname{Im}(A))=\operatorname{rank}(A)=\operatorname{rank}(B) \geq n-1$, and $\operatorname{Im}(A)=Z$. However, since $\prod_{i} y_{i}^{\alpha_{i}}=1$, we have $\sum_{i} \alpha_{i} z_{i}=0$ and $z$ is an element of $Z$. Hence, the system $z=A \mu$ of linear equations has a solution.

B. We show that $B \subset I_{\varphi}(e)$. Take $q_{1}, \ldots, q_{n} \in \mathbb{Q}$. First we show that $\prod_{j=1}^{k} b(j)^{q_{j}} \in$ $I_{\varphi}(e)$ for $k \leq n$. For $k=1$ this follows immediately from Lemma A.4. Now suppose that $\prod_{j=1}^{k} b(j)^{q_{j}} \in I_{\varphi}(e)$. Then, by Lemmas A.1, A.3 and A.4, $\prod_{j=1}^{k+1} b(j)^{q_{j}} \in I_{\varphi}\left(b(k+1)^{q_{k+1}}\right)=I_{\varphi}(e)$. Hence, since $I_{\varphi}(e)$ is closed by Lemma A.2, we obtain $B \subset I_{\varphi}(e)$.

C. From A and B we conclude $H \subset I_{\varphi}(e)$. However, since each ray in $\mathbb{R}_{++}^{n}$ emanating from the origin clearly intersects $H$ exactly once, and also intersects $I_{\varphi}(e)$ exactly once by Lemma 3.1, necessarily $I_{\varphi}(e)=H$.

\section{B Appendix: basic facts for the third characterization}

Let $I$ be a (topologically) closed Abelian group. Let $L, D$ and $R$ be an LDR decomposition of $I$. In this appendix we write the operation $*$ on $I$ as an addition, + , for clarity. ${ }^{21}$ We moreover assume that $I$ is a (closed) subset of $\mathbb{R}^{m}$. It is easy to see that in this case $I$ is a linear subspace of $\mathbb{R}^{m}$ of dimension $n \leq m$. Write $J:=\bar{L} \cap \bar{R}$.

Theorem B.1 Suppose that L is not empty. Then the set $J$ is a closed (and hence linear) subspace of I with $D \subset J$. The dimension of $J$ is either $n$ or $n-1$.

Proof It is straightforward to show that $J$ is a closed linear subspace of $I$. We show that $D \subset J$. Take $d \in D$. Since $L$ is not empty, we can take $x \in L$. Then also $\frac{1}{k} x \in L$ for every natural number $k>0$. So, $d+\frac{1}{k} x \in L$ and $d+\frac{1}{k} x \rightarrow d$ as $k \rightarrow \infty$. Therefore $d \in \bar{L}$. Since $-x \in R$, the same line of reasoning yields $d \in \bar{R}$. Hence, $D \subset J$.

Suppose that the dimension of $J$ is $k<n-1$. We derive a contradiction. Let $T$ be the orthogonal space of $J$ in $I$. Then 0 is the only element of $J$ in $T$; and, since $k<n-1$, the dimension of $T$ is at least 2. So, we can take an $x \in T \backslash J$. Say, $x \in L$. Then $-x \in R \cap T$. However, since the dimension of $T$ is at least 2 , there exists a continuous function $f:[0,1] \rightarrow T \backslash J$ such that $f(0)=x$ and $f(1)=-x$.

Define the image of $f$ by $F:=\{f(\lambda) \mid 0 \leq \lambda \leq 1\}$. Obviously $F$ is path connected. Then, since the sets $F \cap L$ and $F \cap R$ are not empty and form a partition of $F$, there must be a point $y \in F$ that is a cluster point of both $F \cap L$ and $F \cap R$. Such a point is necessarily an element of $J$. Contradiction.

21 Nevertheless, we need the following results only in the context of $I$ as a multiplicative linear space. 
The final result in this appendix refers to the canonical coefficients defined in Sect. 3.3.1.

Lemma B.2 Write $P_{k-1}:=\left\{x \in J_{k-1} \mid \prod_{i} x_{i}^{\beta_{i}^{k}}>1\right\}$. For every $k$ with $2 \leq k \leq K$, either $P_{k-1} \subset L_{\varphi} \cap J_{k-1}$ or $P_{k-1} \subset R_{\varphi} \cap J_{k-1}$.

Proof Suppose this is not the case. Then, since $D_{\varphi} \subset J_{k}$, there is a point $x \in P_{k-1}$ with $x \in L_{\varphi} \cap J_{k-1}$ and a point $y \in P_{k-1}$ with $y \in R_{\varphi} \cap J_{k-1}$. Then there exists a path $^{22} W$ from $x$ to $y$ in $P_{k-1}$ which starts in $L_{\varphi} \cap J_{k-1}$ and ends in $R_{\varphi} \cap J_{k-1}$, while it does not intersect $J_{k}$. This contradicts the definition of $J_{k}$.

\section{References}

Billingsley P (1968) Convergence of probability measures. Wiley series in probability and mathematical statistics, John Wiley \& Sons New York

Birkhoff G (1948) Lattice theory. American Mathematical Society Publications, American Mathematical Society, New York

Conley J, Wilkie S (1996) An extension of the Nash bargaining solution to nonconvex problems. Games Econ Behav 13:26-38

d'Aspremont C (1985) Axioms for social welfare orderings. In: Hurwicz L, Schmeidler D, Sonnenschein $\mathrm{H}$ (eds) Social goals and social organizations. North-Holland, Amsterdam

Debreu G (1964) Continuity properties of Paretian utility. Int Econ Rev 5:285-293

Denicolò V, Mariotti M (2000) Nash bargaining theory, nonconvex problems and social welfare orderings. Theory Decis 48:351-358

Fishburn PC (1972) Even-chance lotteries in social choice theory. Theory Decis 3:18-40

Hamel G (1905) Eine Basis aller Zahlen und die unstetige Lösung der Funktionalgleichung $f(x+y)=$ $f(x)+f(y)$. Math Ann 60:459-462

Harsanyi JC, Selten R (1972) A generalized Nash solution for two-person bargaining games with incomplete information. Manag Sci 18:80-106

Herrero MJ (1989) The Nash program: non-convex bargaining problems. J Econ Theory 49:266-277

Kahneman D, Tversky A (1979) Prospect theory: an analysis of decision under risk. Econometrica 47:263291

Kaneko M (1980) An extension of the Nash bargaining problem and the Nash social welfare function. Theory Decis 12:135-148

Kaneko M, Nakamura K (1979) The Nash social welfare function. Econometrica 47:423-435

Machina MJ (1982) "Expected utility" analysis without the independence axiom. Econometrica 50:277323

Marek VW, Mycielski J (2001) Foundations of mathematics in the twentieth century. Am Math Month 108:449-468

Mariotti M (1998a) Nash bargaining theory when the number of alternatives can be finite. Soc Choice Welf $15: 413-421$

Mariotti M (1998b) Extending Nash's axioms to nonconvex problems. Games Econ Behav 22:377-383

Maschler M, Owen G, Peleg B (1988) Paths leading to the Nash set. In: Roth AE (ed) The shapley value: essays in honor of Lloyd S. Shapley. Cambridge University Press, Cambridge UK, pp 321-330

Mycielski J, Swierczkowski S (1964) On the Lebesgue measurability and the Axiom of Determinateness. Fundam Math 54:67-71

Nash JF (1950) The bargaining problem. Econometrica 18:155-162

Naumova N, Yanovskaya E (2001) Nash social welfare orderings. Math Soc Sci 42:203-231

Podnieks K (2007) http://www.ltn.lv/ podnieks/gt.html

Roth AE (1977) Individual rationality and Nash's solution to the bargaining problem. Math Oper Res 2: $64-65$

Shubik M (1982) Game theory in the social sciences: concepts and solutions. MIT Press, Cambridge

$\overline{22}$ A set $W$ is called a path if there exists an onto continuous function $f:[0,1] \rightarrow W$. 
Starmer C (2000) Developments in non-expected utility theory: the hunt for a descriptive theory of choice under risk. J Econ Lit 38:332-382

$\mathrm{Xu}$ Y, Yoshihara N (2006) Alternative characterizations of three bargaining solutions for nonconvex bargaining problems. Games Econ Behav 57:86-92

Zame WR (2007) Can intergenerational equity be operationalized. Theor Econ 2:187-202

Zhou L (1996) The Nash bargaining theory with non-convex problems. Econometrica 65:681-685 